\title{
The neon content of nearby B-type stars and its implications for the solar model problem $\star, \star \star$
}

\author{
T. Morel ${ }^{1,2}$ and K. Butler ${ }^{3}$ \\ ${ }^{1}$ Institut d'Astrophysique et de Géophysique, Université de Liège, Allée du 6 août, Bât. B5c, 4000 Liège, Belgium \\ e-mail: morel@astro.ulg.ac.be \\ 2 Katholieke Universiteit Leuven, Departement Natuurkunde en Sterrenkunde, Instituut voor Sterrenkunde, Celestijnenlaan 200D, \\ 3001 Leuven, Belgium \\ 3 Universitäts-Sternwarte München, Scheinerstrasse 1, 81679 München, Germany
}

Received 7 April 2008 / Accepted 28 May 2008

\begin{abstract}
The recent downward revision of the solar photospheric abundances now leads to severe inconsistencies between the theoretical predictions for the internal structure of the Sun and the results of helioseismology. There have been claims that the solar neon abundance may be underestimated and that an increase in this poorly-known quantity could alleviate (or even completely solve) this problem. Early-type stars in the solar neighbourhood are well-suited to testing this hypothesis because they are the only stellar objects whose absolute neon abundance can be derived from the direct analysis of photospheric lines. Here we present a fully homogeneous NLTE abundance study of the optical Ne I and Ne II lines in a sample of 18 nearby, early B-type stars, which suggests $\log \epsilon(\mathrm{Ne})=7.97 \pm 0.07 \mathrm{dex}$ (on the scale in which $\log \epsilon[\mathrm{H}]=12$ ) for the present-day neon abundance of the local interstellar medium (ISM). Chemical evolution models of the Galaxy only predict a very small enrichment of the nearby interstellar gas in neon over the past $4.6 \mathrm{Gyr}$, implying that our estimate should be representative of the Sun at birth. Although higher by about 35\% than the new recommended solar abundance, such a value appears insufficient by itself to restore the past agreement between the solar models and the helioseismological constraints.
\end{abstract}

Key words. stars: early-type - stars: fundamental parameters - stars: abundances - stars: atmospheres - Sun: helioseismology

\section{Introduction}

State-of-the-art spectral analyses of solar photospheric lines using time-dependent, 3-D hydrodynamical models (Asplund et al. 2005, and references therein, hereafter AGS05) have recently led to a reduction of the commonly accepted abundances of the dominant metals in the Sun (Grevesse \& Sauval 1998, hereafter GS98). This, in turn, greatly affects the input physics of the standard solar models (e.g. radiative opacities) and considerably worsens the agreement between the theoretical predictions and the results of helioseismic inversions. In particular, the sound speed and density profiles in the solar interior are no longer well reproduced, while the convective zone is predicted to be too shallow and with a helium abundance that is too low (see, e.g. Basu \& Antia 2008, for a comprehensive review and an account of the various solutions proposed to solve this problem).

Neon is one of the most important contributors to the opacity at the base of the convective zone, after oxygen and iron. Contrary to these latter two elements whose abundance can be estimated from the analysis of photospheric lines (or is even accurately known from meteoritic data in the case of $\mathrm{Fe}$ ), the $\mathrm{Ne}$ abundance is not well constrained. As noble gases are not retained in CI chondrite meteorites and Ne I lines are completely lacking in the solar spectrum because of their high excitation

\footnotetext{
* Appendices A and B are only available in electronic form at http://www . aanda. org

$\star \star$ Table B.1 is also available in electronic form at the CDS via anonymous ftp to cdsarc.u-strasbg.fr $(130.79 .128 .5)$ or via http://cdsweb.u-strasbg.fr/cgi-bin/qcat?J/A+A/487/307
}

energies, one has to rely instead on indirect estimates based on observations of coronal lines or high-energy particles, which are by themselves prone to large uncertainties (see below). The $\mathrm{Ne}$ abundance of the Sun is usually based on measurements of the $[\mathrm{Ne} / \mathrm{O}]$ abundance ratio in the solar upper atmosphere and has been scaled down (by 0.17 dex) to account for the decrease in the solar oxygen content (an additional correction amounting to -0.07 dex arises from the adoption of a different neonto-oxygen abundance ratio, as determined from energetic particles; Reames 1999). This leads to a value lowered from 8.08 (GS98) to 7.84 dex (AGS05). An upward revision of this uncertain quantity has therefore been invoked as a possible way to compensate for the decrease in opacity brought about by the lower abundances of the other chemical elements. Standard, full solar models constructed with different chemical mixtures suggest that an increase of the $\mathrm{Ne}$ abundance by $0.4-0.5 \mathrm{dex}$, along with a possible adjustment of the other metal abundances within their uncertainties, is required (Bahcall et al. 2005). Models with values outside this range do not simultaneously reproduce the helioseismic constraints that are the He abundance/depth of the convective zone and the sound-speed/density profiles in the interior (see also Delahaye \& Pinsonneault 2006). It was also shown that an increase of this magnitude provides a better match to the properties of the solar core, as probed by low-degree $p$ modes (Basu et al. 2007; Zaatri et al. 2007). Observations of a large sample of active stars with the Chandra X-ray observatory indeed seemed at first sight to support an upward revision of the newly adopted solar $\mathrm{Ne}$ abundance at the required levels (Drake \& Testa 2005), but much lower values were subsequently 
Table 1. Program stars and spectroscopic data used.

\begin{tabular}{|c|c|c|c|c|c|c|}
\hline $\begin{array}{c}\text { HD } \\
\text { Number }\end{array}$ & $\begin{array}{l}\text { Alternative } \\
\text { ID }\end{array}$ & $\operatorname{Remarks}^{a}$ & Telescope & Instrument & $\begin{array}{c}\text { Dates of } \\
\text { observation }\end{array}$ & $\begin{array}{l}\text { Number of } \\
\text { exposures }\end{array}$ \\
\hline 886 & $\gamma$ Peg & SB1, $\beta$ Cephei & 1.9-m OHP & ELODIE & 1998-2004 & 47 \\
\hline 16582 & $\delta$ Cet & $\beta$ Cephei & 1.2-m ESO/Euler & CORALIE & July 2002 & 4 \\
\hline 29248 & $v$ Eri & $\beta$ Cephei & 1.2-m ESO/Euler & CORALIE & 2001-2002 & 579 \\
\hline 30836 & $\pi^{4}$ Ori & SB1 & 1.2-m ESO/Euler & CORALIE & March 2007 & 4 \\
\hline 35468 & $\gamma$ Ori & $\ldots$ & 1.9-m OHP & ELODIE & January 2003 & 2 \\
\hline 36591 & $\ldots$ & $\ldots$ & 1.9-m OHP & ELODIE & November 2004 & 1 \\
\hline 44743 & $\beta \mathrm{CMa}$ & $\beta$ Cephei & 1.2-m ESO/Euler & CORALIE & 2000-2004 & 449 \\
\hline 46328 & $\xi^{1} \mathrm{CMa}$ & $\beta$ Cephei & 1.2-m ESO/Euler & CORALIE & 2000-2004 & 79 \\
\hline 50707 & $15 \mathrm{CMa}$ & $\beta$ Cephei & 1.2-m ESO/Euler & CORALIE & March 2007 & 3 \\
\hline 51309 & $\iota \mathrm{CMa}$ & $\ldots$ & 2.2-m ESO/MPG & FEROS & April 2005 & 6 \\
\hline 52089 & $\epsilon \mathrm{CMa}$ & $\ldots$ & 2.2-m ESO/MPG & FEROS & April 2005 & 4 \\
\hline 129929 & V836 Cen & $\beta$ Cephei & 2.2-m ESO/MPG & FEROS & May 2003 & 1 \\
\hline 149438 & $\tau$ Sco & $\ldots$ & 1.2-m ESO/Euler & CORALIE & March 2007 & 2 \\
\hline 163472 & V2052 Oph & $\beta$ Cephei & 2.7-m McDonald & CS2 & July 2004 & 105 \\
\hline 170580 & $\ldots$ & $\ldots$ & 1.5-m ESO & FEROS & July 2001 & 1 \\
\hline 180642 & $\ldots$ & $\beta$ Cephei & 2.2-m ESO/MPG & FEROS & May 2006 & 11 \\
\hline 205021 & $\beta$ Сер & SB1, $\beta$ Cephei & 1.9-m OHP & ELODIE & 1995-2003 & 28 \\
\hline 214993 & $12 \mathrm{Lac}$ & $\beta$ Cephei & 2.7-m McDonald & CS2 & July 2004 & 31 \\
\hline
\end{tabular}

Notes: The ELODIE spectra have been extracted from the instrument archives (see http://atlas.obs-hp.fr/elodie/). The mean resolving power of the spectrographs amounts to about $R \sim 50000$, except in the case of CS2 $R \sim 60000)$. The signal-to-noise ratio in the individual exposures typically lies in the range 150-200. Further details on these observations can be found in Morel et al. (2006, 2008), Morel \& Aerts (2007), Hubrig et al. (2008), and references therein.

${ }^{a}$ We only consider as $\beta$ Cephei stars the confirmed candidates in the recent catalogue of Stankov \& Handler (2005).

inferred for solar-like stars (e.g. Liefke \& Schmitt 2006) or quiescent solar regions (e.g. Young 2005), thus leaving this question still open.

The abundance patterns observed in stellar coronae appear at odds with the solar mixture, in particular, with neon being strongly enhanced in active stars by some still poorly-understood mechanisms (e.g. Drake et al. 2001). An attractive alternative to constrain the neon content of the Sun is, however, offered by the direct analysis of Ne I and Ne II photospheric lines in nearby OB stars. Although neon has traditionally been largely neglected in past abundance studies of massive stars, this "solar model crisis" has indeed renewed interest in determining the abundance of this element in nearby objects. Since the publication of AGS05 results, however, only a single study addressing this issue appeared in the literature (Cunha et al. 2006). A relatively high mean Ne abundance was found in a sample of 11 B-type dwarfs in the Orion association ( 0.27 dex above AGS05 value), but this still falls short (by a factor $\sim 1.5$ ) of completely solving the controversy discussed above. To shed more light on this issue, here we present a fully homogeneous NLTE abundance analysis of a sample of 18 early B-type stars in the solar neighbourhood. The results presented in this paper supersede previous preliminary reports (Morel \& Butler 2007, 2008), as significant improvements in the model atom have been made during this interval.

\section{Program stars and observational material}

The list of the program stars is provided in Table 1, along with the source of the high-resolution spectroscopic data used. Independent pieces of evidence have been presented for a Galactic abundance gradient for $\mathrm{Ne}$, either from observations of B stars (Kilian-Montenbruck et al. 1994), planetary nebulae (e.g. Henry et al. 2004) or H II regions (e.g. Martín-Hernández et al. 2002). Therefore, we only selected stars within $\sim 1 \mathrm{kpc}$, as estimated from Hipparcos parallaxes or open cluster membership.
These stars are also uniformly distributed in terms of Galactic longitude, ensuring that the abundances we derive truly reflect those of the local ISM. The vast majority of our targets have effective temperatures lying in the range $21000-28000 \mathrm{~K}$. Based on our own calculations, the $\mathrm{Ne}$ I and $\mathrm{Ne}$ II lines reach a maximum strength around $T_{\text {eff }} \sim 17000$ and $\sim 31000 \mathrm{~K}$, respectively. Although the diagnostic lines will be weak, our targets therefore sample a crucial $T_{\text {eff }}$ range where lines of both ions are measurable and can be independently used to determine the neon abundance. As will be shown below, forcing agreement between the mean abundances provided by both ions allows one to correct for the existence of systematic errors in the data (e.g. imperfect temperature scale).

Our sample comprises several pulsating B-type stars of the $\beta$ Cephei class (Table 1). In many cases, these objects have been the targets of intensive spectroscopic monitoring campaigns dedicated to a study of their pulsational behaviour (e.g. Aerts et al. 2004; Mazumdar et al. 2006). We make use of these data here and base our analysis in these cases on a mean spectrum created by averaging the whole set of individual exposures (all spectra were put in the laboratory rest frame prior to this operation). Using such a large (up to 579) number of time-resolved spectra not only ensures that the derived atmospheric parameters are representative of the values averaged over the pulsation cycle, but also allows us to measure with reasonable confidence some extremely weak lines with equivalent widths $(E W \mathrm{~s})$ down to only a few $m \AA$ (see Table B.1). The accuracy of these measurements is discussed in Sect. 3.

Three stars in our sample are known single-lined binaries (Table 1). The companions to $\gamma$ Peg and $\beta$ Cep are both very faint and the contamination of the primary spectrum can be neglected (Roberts et al. 2007; Schnerr et al. 2006). In the case of $\pi^{4}$ Ori (Luyten 1936), the lack of information about the secondary forced us to treat it as a single star (Gies \& Lambert 1992 classify this system as B2 III + B2 IV, but no details are given). 


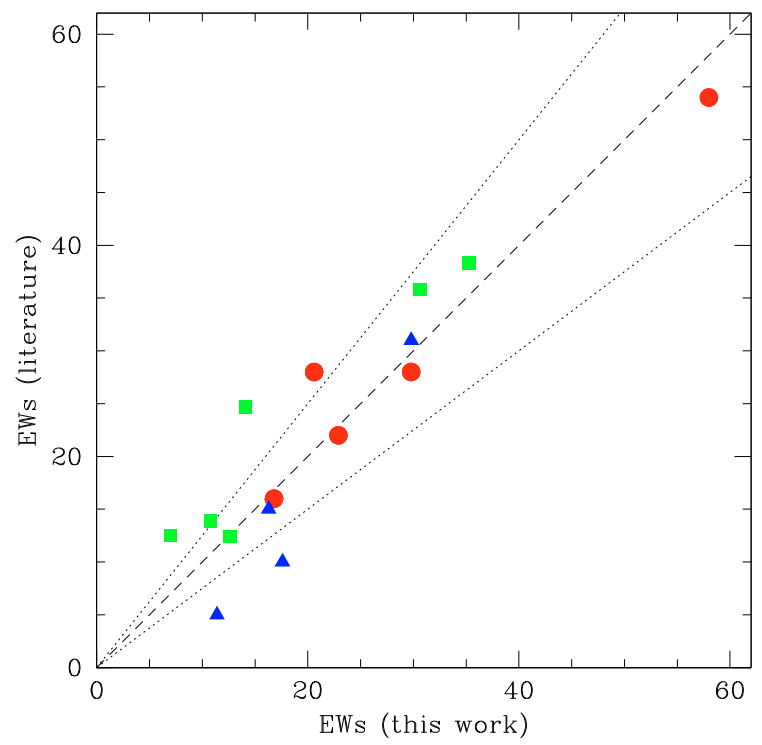

Fig. 1. Comparison between our measured $E W \mathrm{~s}$ and values in the literature. Circles: Aller \& Jugaku (1958), but excluding uncertain values, triangles: Gies \& Lambert (1992), squares: Kilian \& Nissen (1989). The 1:1 relationship is shown as a dashed line, while the two dotted lines indicate a discrepancy reaching $25 \%$.

\section{Methods of analysis}

A standard, iterative scheme is used to self-consistently derive the atmospheric parameters purely on spectroscopic grounds: the effective temperature $\left(T_{\text {eff }}\right)$ is determined from the $\mathrm{Si} \mathrm{II/III/IV}$ ionization balance, the logarithmic surface gravity $(\log g)$ from fitting the collisionally-broadened wings of the Balmer lines and the microturbulent velocity $(\xi)$ from the $\mathrm{O}$ II features requiring that the abundances yielded are independent of line strength. It must be emphasised that our analysis is fully consistent, as the methodology used to derive the atmospheric parameters and elemental abundances is identical in all respects for all stars.

Our targets are all narrow lined $\left(v \sin i \lesssim 65 \mathrm{~km} \mathrm{~s}^{-1}\right)$ and classical curve-of-growth techniques were used to derive the neon abundances using the $E W \mathrm{~s}$ of a set of $\mathrm{Ne}$ I/II features selected as being unblended in the relevant temperature range. The diagnostic lines were chosen after careful inspection of spectral atlases based on the extensive line list of Kurucz \& Bell $(1995)^{1}$ and the $E W \mathrm{~s}$ were measured by direct integration. Our final line list is made up of $12 \mathrm{Ne}$ I and $8 \mathrm{Ne}$ II lines, and our results are based on 2 to 18 lines for a given star (see Table B.1). To roughly assess the accuracy of our $E W \mathrm{~s}$, we have selected a few lines of various strengths and repeated the measurements for the 79 individual exposures obtained for $\xi^{1} \mathrm{CMa}$. The dispersion of the results was of the order of 30,15 and $10 \%$ for features with $E W=5,10$ and $20 \mathrm{~m} \AA$, respectively. This may be taken as the typical accuracy for stars with only a few spectra available, while significantly lower values are likely for stars with a very high-quality mean spectrum (see Table 1). A comparison with the few measurements in the literature independently supports these order-of-magnitude estimates: a disagreement reaching on average about 35 and $10 \%$ is observed for lines with $E W$ s lower or greater than $20 \mathrm{~m} \AA$, respectively (Fig. 1).

To obtain the NLTE abundances, we made use of the latest versions of the line formation codes DETAIL/SURFACE and plane-parallel, fully line-blanketed, LTE atmospheric models

\footnotetext{
${ }^{1}$ See the online spectral atlases of main-sequence B stars at: http: // www.lsw. uni-heidelberg.de/cgi-bin/websynspec.cgi
}

(Kurucz 1993). We adopted models with $\mathrm{He} / \mathrm{H}=0.089$ by number in all cases. The only exception was V2052 Oph, as this star displays some evidence for a helium enrichment (Morel et al. 2006; Neiner et al. 2003). However, adopting a solar helium abundance in that case would have a negligible impact on the resulting Ne abundance: $\Delta \log \epsilon(\mathrm{Ne}) \lessgtr 0.01$ dex.

We have developed an extensive model atom consisting of 153, 78 and 5 levels for Ne I, Ne II and Ne III, respectively (along with the ground state of $\mathrm{Ne}$ IV) ${ }^{2}$.

The energy levels are those of Saloman \& Sansonetti (2004) for Ne I, Kramida \& Nave (2006a) and Kramida et al. (2006) for Ne II, and Kramida \& Nave (2006b) for Ne III, as tabulated on the NIST web site (Ralchenko et al. 2008). The fine structure data of Ne II and Ne III have been combined according to the LS-coupling scheme, while fine structure has been retained for Ne I. Oscillator strengths and photoionization cross sections for Ne I and Ne II are the result of Breit-Pauli R-matrix (BPRM, Hummer et al. 1993) calculations (Butler 2008a,b), while those for Ne III are from an LS-coupling R-matrix computation (Butler 2008c).

A further BPRM calculation (Butler 2008d) has provided collisional data for the levels up to $5 \mathrm{f}$ of Ne I. Griffin et al. (2001) performed a 61 term, 138 level, Intermediate-Coupling FrameTransformation (ICFT) R-matrix calculation for Ne II. These data provide accurate collisional rates for the majority of the $\mathrm{Ne}$ II terms considered. All remaining bound-bound collisional rates were estimated, either using the van Regemorter (1982) approximation for the allowed transitions or assuming a constant effective collision strength $(\Upsilon=1)$ for the forbidden ones. All collisional ionization rates made use of the Seaton (1962) approximation in which the photoionization cross sections at threshold were taken from the respective R-matrix results.

Since intermediate oscillator strengths are available, we have used these directly in the spectral synthesis. The damping constants needed were obtained from the R-matrix data (radiative) and from the prescription of Cowley (1971, collisional).

Although we recall that our abundance results solely rely on $E W$ measurements and are not based on line-profile fitting techniques, the observed (weak) Ne features are well reproduced within the noise limits in all but a few cases (see Figs. A.1-A.4).

\section{Abundance results}

The Ne I lines in B-type stars are known to suffer considerable NLTE strengthening (as first demonstrated by Auer \& Mihalas 1973), leading to spuriously high LTE abundances (e.g. Gies \& Lambert 1992; Martin 2004). The NLTE corrections are much less severe in the case of the Ne II lines, however, with values typically amounting to only about -0.05 dex in our program stars, compared to -0.40 dex for the Ne I lines. No strong starto-star variations in the magnitude of these corrections are seen within our sample, either for Ne I or Ne II (see Fig. 2). The departures from LTE for the Ne I lines are found to increase with the line strength (note that the strong trend between the reduced $E W \mathrm{~s}$ and the abundances is removed under the assumption of NLTE) and reach a maximum for Ne I 26402 . As Cunha et al. (2006), we find a small decrease of the NLTE corrections with increasing temperature for this transition, but our values appear to be systematically slightly larger (by $\sim 0.1 \mathrm{dex}$ ).

Using the $T_{\text {eff }}$ values derived from the silicon ionization balance, we find a slight, albeit clearly significant

2 The model atoms used are available from the authors on request. 


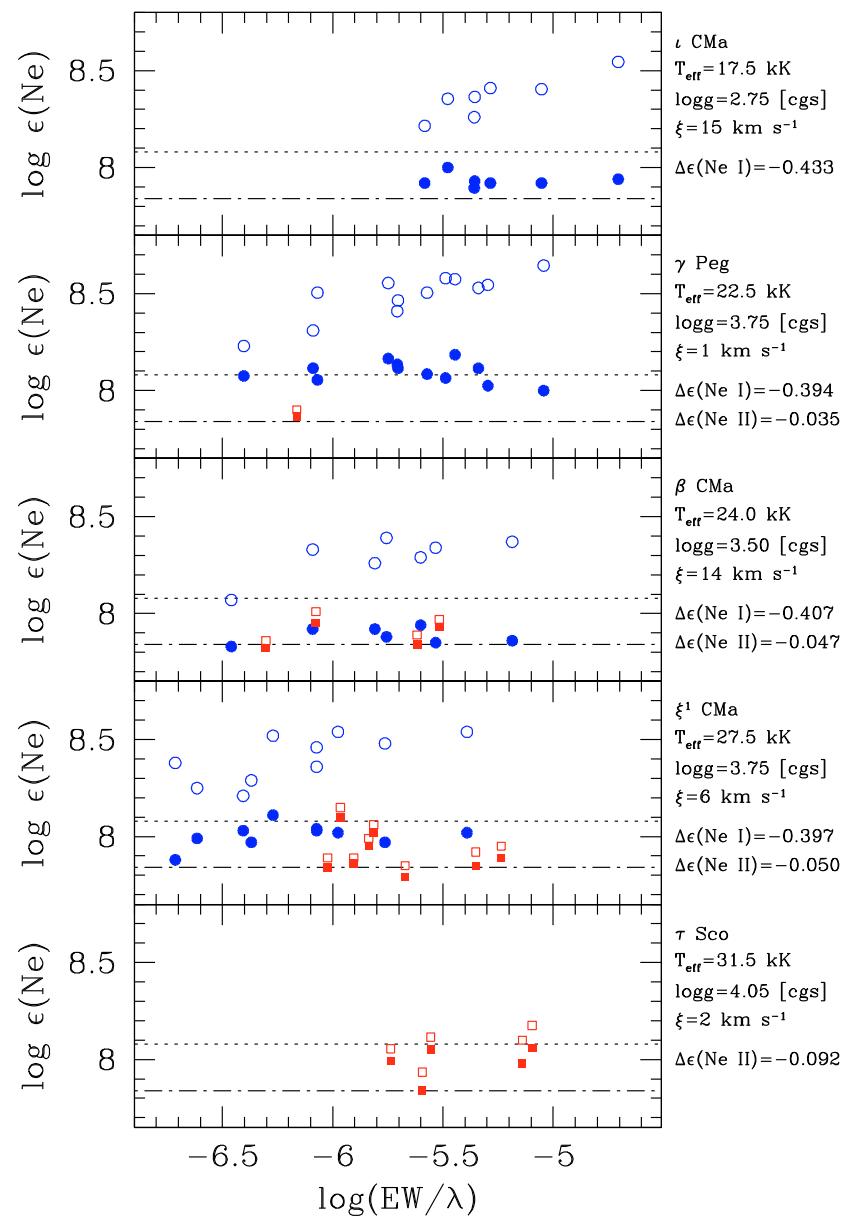

Fig. 2. Variations of the LTE (open symbols) and NLTE (filled symbols) abundances, as a function of the reduced $E W$ s. The Ne I and Ne II lines are denoted by circles and squares, respectively (in the online version of this journal, these are plotted in blue and red, respectively). The dotted and dashed-dotted lines indicate the solar Ne abundances of GS98 and AGS05, respectively. Each panel corresponds to a different star (ordered from top to bottom by increasing $T_{\text {eff }}$ ). The adopted physical parameters and mean NLTE corrections for the Ne I and Ne II lines, $\Delta \epsilon=\log (\epsilon)_{\mathrm{NLTE}}-\log (\epsilon)_{\mathrm{LTE}}$ (in dex), are given on the right-hand side of the panels.

difference $(0.17$ dex $)$ between the mean Ne I and Ne II abundances (Fig. 3, left-hand panels). We mainly attribute this discrepancy to uncertainties in the temperature scale and not to inaccuracies in the NLTE corrections or line formation treatment. To remedy this problem, we therefore re-determined the $T_{\text {eff }}$ values for the 11 stars with abundances estimated both from $\mathrm{Ne}$ I and $\mathrm{Ne}$ II by imposing $\mathrm{Ne}$ ionization balance. This leads to a slightly cooler temperature scale with an average downward revision typical of the uncertainties and amounting to $825 \mathrm{~K}$ $(\sim 3.5 \%)$. This mean value was subsequently used to rescale the effective temperatures for the 7 remaining stars with only Ne I or Ne II lines measured. As the determinations of $T_{\text {eff }}$ and $\log g$ are coupled, and to obtain similar quality fits to the wings of the Balmer lines, we also lowered the $\log g$ values accordingly (the microturbulent velocity was kept unchanged). The default and revised atmospheric parameters are given in Table 2, while the corresponding abundances are provided in Table 3 . The quoted uncertainties take into account both the line-to-line scatter ${ }^{3}$ and

${ }^{3}$ In case where the Ne II abundance is based on a single line, we adopted a typical value of 0.10 dex. the errors arising from the uncertainties on the atmospheric parameters (see Morel et al. 2006). Table 4 illustrates these calculations in the case of $\beta \mathrm{CMa}$. The uncertainties affecting the Ne II abundances are quite large for the coolest objects in which lines of this ion could be measured (e.g. $\gamma$ Peg; Table 3 ) because of the strong sensitivity of the results to errors on $T_{\text {eff. }}$. We regard the abundances obtained using the revised effective temperatures as the most reliable and will only discuss these values in the following, but we emphasize that the choice of the $T_{\text {eff }}$ scale does not affect the conclusions drawn in this paper. As can be seen in Fig. 3, the mean $\mathrm{Ne}$ abundances derived using all lines only differ by 0.03 dex. Although our sample spans a wide range in terms of temperature $\left(\Delta T_{\mathrm{eff}} \sim 14000 \mathrm{~K}\right)$, no trend between the abundance data (either Ne I or Ne II) and $T_{\text {eff }}$ is apparent (Fig. 3, right-hand panels). The small scatter observed (standard deviation of 0.07 dex) can be explained by the measurements uncertainties alone and indicates a well-mixed local ISM with a homogeneous Ne abundance.

\section{Discussion}

\subsection{Comparison with previous results for B-type stars}

A mean neon abundance, $\log \epsilon(\mathrm{Ne})=7.97 \pm 0.07 \mathrm{dex}$, is inferred from our combined NLTE abundance analysis of the Ne I and Ne II lines (a nearly identical result to within 0.01 dex is obtained when weighting the individual abundances by their respective uncertainties). We compare below our results with those reported in the literature from the NLTE analysis of the Ne I lines. To our knowledge, only a single study based on Ne II has been performed (Kilian 1994). Although LTE was assumed, these results will also be discussed in the following in view of the smallness of the NLTE corrections for this ion (Sect. 4).

First, a significantly higher value, $\log \epsilon(\mathrm{Ne})=8.11 \pm$ 0.04 dex, has recently been reported from the analysis of $8 \mathrm{Ne}$ I transitions in 11 Orion B-type dwarfs by Cunha et al. (2006) using an extensive TLUSTY model atom and NLTE model atmospheres (Hubeny \& Lanz 1995). The adopted $T_{\text {eff }}$ and $\log g$ values were taken from Cunha \& Lambert (1992). The effective temperatures were estimated from Strömgren indices and the surface gravities from fitting the collisionally-broadened wings of $\mathrm{H} \gamma$. One star (HD 36591) is common to both studies and follows this general trend, as we obtain a nominal value lower by 0.14 dex. This discrepancy may have various causes and can stem from: (a) the magnitude of the applied NLTE corrections: as mentioned in Sect. 4, and although this conclusion is only based on a single transition, there is some indication that ours are slightly larger; (b) differences in the analytical procedures used to derive the atmospheric parameters ${ }^{4}$; (c) the choice of the atomic data: Table 5 shows, however, that our $\log g f$ values are in good agreement with theirs (taken from Seaton 1998); or (d) the type of model atmosphere employed (LTE vs NLTE). Nieva \& Przybilla (2007) carried out a thorough comparison in the case of $\mathrm{OB}$ dwarfs and giants between the model predictions

\footnotetext{
${ }^{4}$ We note that some of their adopted surface gravities are uncomfortably large: up to $\log g=4.70$ dex in the complete sample analysed by Cunha \& Lambert (1992) and up to $\log g=4.41 \mathrm{dex}$ in the sample of Cunha et al. (2006). The atmospheric parameters obtained for HD 36591 are, however, in reasonable agreement: $T_{\text {eff }}=$ $27000 \pm 1000 \mathrm{~K}$ and $\log g=4.00 \pm 0.20 \mathrm{dex}$ (this study) against $T_{\text {eff }}=26330 \pm 750 \mathrm{~K}$ and $\log g=4.21 \pm 0.10 \mathrm{dex}$ (Cunha et al. 2006). Our microturbulent velocity $\left(\xi=3 \pm 2 \mathrm{~km} \mathrm{~s}^{-1}\right)$ is very close to their fixed value, $\xi=5 \mathrm{~km} \mathrm{~s}^{-1}$, but the $\mathrm{Ne}$ abundances are, in any case, largely insensitive to this quantity (Table 4 ).
} 


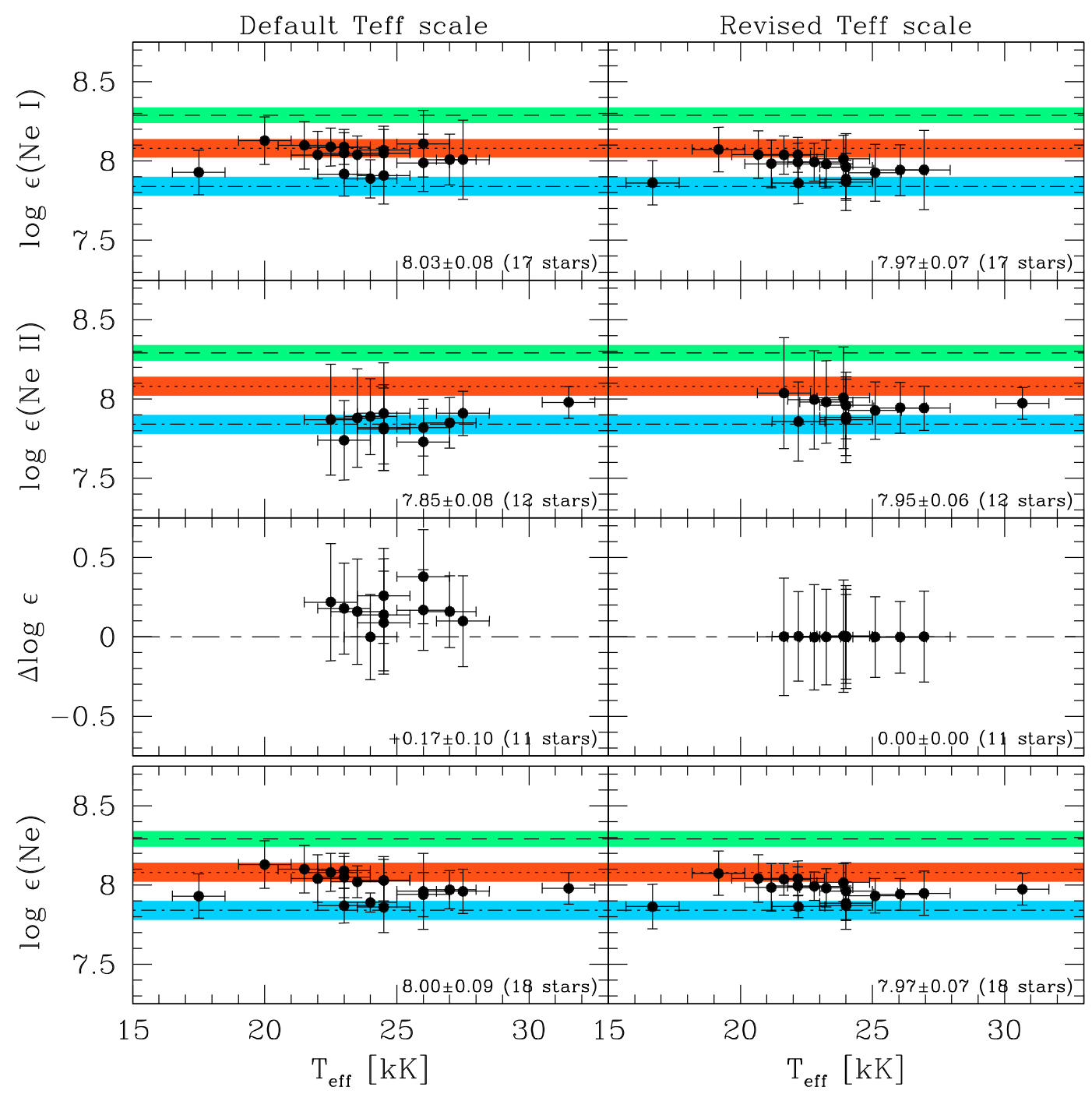

Fig. 3. Abundance data as a function of the effective temperature for the default (left panels) and the revised (right panels) temperature scale (see Sect. 4). From top to bottom: variation of the Ne I abundances, the Ne II abundances, the difference between the abundances yielded by the Ne I and $\mathrm{Ne}$ II lines, and the mean neon abundance using lines of both ions. The unweighted mean data value is given in each panel. The long-dashed line indicates the neon abundance needed to reconcile the solar interior models and the results of helioseismology $(8.29 \pm 0.05$ dex; Bahcall et al. 2005), while the dotted and dashed-dotted lines show the standard (8.08 \pm 0.06 dex; GS98) and the new (7.84 \pm 0.06 dex; AGS05) solar Ne abundances, respectively. The horizontal, shaded strips show the corresponding uncertainties. In the online version of this journal, these are plotted in green, red and blue, respectively.

obtained assuming line-blanketed, LTE model atmospheres coupled with a detailed NLTE line-formation treatment (as in our study) or a self-consistent, full NLTE approach (as in Cunha et al. 2006). Excellent agreement was found for $\tau$ Sco both for the atmospheric structure (e.g. temperatures deviating by less than $1 \%$ in the Ne line-formation regions) and for the theoretical $\mathrm{H}$ and $\mathrm{He}$ line profiles, which were found to be nearly indistinguishable when using identical model atoms. Even smaller discrepancies are expected for the other stars in our sample, as NLTE effects are very likely to be less critical in cooler dwarfs. Although detailed calculations (beyond the scope of this paper) are needed to quantitatively examine this issue, this supports the idea that the hybrid approach adopted here is suitable for an accurate determination of $\mathrm{Ne}$ abundances in $\mathrm{B}$ dwarfs at solar metallicity (and additionally much less demanding in terms of computer resources).

An NLTE neon abundance study of 9 bright B5-B9 stars was also presented by Hempel \& Holweger (2003). After deriving $T_{\mathrm{eff}}$ and $\log g$ from photometric data, they obtained $\log \epsilon(\mathrm{Ne})=8.16 \pm 0.14 \mathrm{dex}$ from the Ne I $\lambda 6402$ and Ne I $\lambda 6506$ lines using a model atom consisting of 45 and 47 levels for $\mathrm{Ne}$ I and Ne II, respectively.

Using a 31-level Ne I model atom and TLUSTY NLTE model atmospheres, Dworetsky \& Budaj (2000) obtained $\log \epsilon(\mathrm{Ne})=8.10 \pm 0.09$ dex for 7 late B/early A stars based on the modelling of $\mathrm{Ne}$ I $\lambda 6402$. The atmospheric parameters were estimated from a combination of photometric and spectroscopic techniques.

On the other hand, Sigut (1999) developed a model atom consisting of $37 \mathrm{Ne}$ I and $11 \mathrm{Ne}$ II levels to recompute the NLTE abundances of 14 early B-type stars in the sample of Gies \& Lambert (1992) using their $E W$ s for Ne I $\lambda 6506$ and adopted atmospheric parameters. A mean value, $\log \epsilon(\mathrm{Ne}) \sim 8.12 \mathrm{dex}$, was obtained, but the scatter is very large (spread of $0.7 \mathrm{dex}$ ). Furthermore, the $T_{\text {eff }}$ scale assumed by Gies \& Lambert (1992) is likely too hot (see discussion in Lyubimkov et al. 2002).

Finally, Kilian (1994) derived the $\mathrm{Ne}$ abundance of 12 nearby, early B-type stars using a set of $\mathrm{Ne}$ II lines and found 
Table 2. Default and adopted atmospheric parameters with $T_{\text {eff }}$ estimated from the Si and Ne ionization balance, respectively (see Sect. 4).

\begin{tabular}{|c|c|c|c|c|c|c|c|c|c|}
\hline \multirow[b]{2}{*}{$\begin{array}{c}\text { HD } \\
\text { Number }\end{array}$} & \multirow[b]{2}{*}{$\begin{array}{l}\text { Alternative } \\
\text { ID }\end{array}$} & \multicolumn{4}{|c|}{ Default $T_{\text {eff }}$ Scale } & \multicolumn{4}{|c|}{ Revised $T_{\text {eff }}$ Scale } \\
\hline & & $\begin{array}{l}T_{\text {eff }} \\
(\mathrm{K})\end{array}$ & $\begin{array}{c}\log g \\
\left(\mathrm{~cm} \mathrm{~s}^{-2}\right)\end{array}$ & $\begin{array}{c}\xi \\
\left(\mathrm{km} \mathrm{s}^{-1}\right)\end{array}$ & Ref. & $\begin{array}{l}T_{\text {eff }} \\
(\mathrm{K})\end{array}$ & $\begin{array}{c}\log g \\
\left(\mathrm{~cm} \mathrm{~s}^{-2}\right)\end{array}$ & $\begin{array}{c}\xi \\
\left(\mathrm{km} \mathrm{s}^{-1}\right)\end{array}$ & Ref. \\
\hline 886 & $\gamma$ Peg & 22500 & $3.75 \pm 0.15$ & $1_{-1}^{+2}$ & 1 & 21650 & $3.67 \pm 0.15$ & $1_{-1}^{+2}$ & 5 \\
\hline 16582 & $\delta$ Cet & 23000 & $3.80 \pm 0.15$ & $1_{-1}^{+3}$ & 1 & 22175 & $3.72 \pm 0.15$ & $1_{-1}^{+3}$ & 5 \\
\hline 29248 & $v$ Eri & 23500 & $3.75 \pm 0.15$ & $10 \pm 4$ & 1 & 22800 & $3.68 \pm 0.15$ & $10 \pm 4$ & 5 \\
\hline 30836 & $\pi^{4}$ Ori & 21500 & $3.35 \pm 0.15$ & $8 \pm 4$ & 2 & 20675 & $3.27 \pm 0.15$ & $8 \pm 4$ & 5 \\
\hline 35468 & $\gamma$ Ori & 22000 & $3.50 \pm 0.20$ & $13 \pm 5$ & 2 & 21175 & $3.42 \pm 0.20$ & $13 \pm 5$ & 5 \\
\hline 36591 & $\ldots$ & 27000 & $4.00 \pm 0.20$ & $3 \pm 2$ & 2 & 26050 & $3.91 \pm 0.20$ & $3 \pm 2$ & 5 \\
\hline 44743 & $\beta \mathrm{CMa}$ & 24000 & $3.50 \pm 0.15$ & $14 \pm 3$ & 1 & 24000 & $3.50 \pm 0.15$ & $14 \pm 3$ & 5 \\
\hline 46328 & $\xi^{1} \mathrm{CMa}$ & 27500 & $3.75 \pm 0.15$ & $6 \pm 2$ & 1 & 26950 & $3.70 \pm 0.15$ & $6 \pm 2$ & 5 \\
\hline 50707 & $15 \mathrm{CMa}$ & 26000 & $3.60 \pm 0.15$ & $7 \pm 3$ & 2 & 24000 & $3.40 \pm 0.15$ & $7 \pm 3$ & 5 \\
\hline 51309 & $\iota \mathrm{CMa}$ & 17500 & $2.75 \pm 0.15$ & $15 \pm 5$ & 2 & 16675 & $2.67 \pm 0.15$ & $15 \pm 5$ & 5 \\
\hline 52089 & $\epsilon \mathrm{CMa}$ & 23000 & $3.30 \pm 0.15$ & $16 \pm 4$ & 2 & 22200 & $3.22 \pm 0.15$ & $16 \pm 4$ & 5 \\
\hline 129929 & V836 Cen & 24500 & $3.95 \pm 0.20$ & $6 \pm 3$ & 1 & 23900 & $3.89 \pm 0.20$ & $6 \pm 3$ & 5 \\
\hline 149438 & $\tau$ Sco & 31500 & $4.05 \pm 0.15$ & $2 \pm 2$ & 3 & 30675 & $3.97 \pm 0.15$ & $2 \pm 2$ & 5 \\
\hline 163472 & V2052 Oph & 23000 & $4.00 \pm 0.20$ & $1_{-1}^{+4}$ & 1 & 22175 & $3.92 \pm 0.20$ & $1_{-1}^{+4}$ & 5 \\
\hline 170580 & $\ldots$ & 20000 & $4.10 \pm 0.15$ & $1_{-1}^{+5}$ & 4 & 19175 & $4.02 \pm 0.15$ & $1_{-1}^{+5}$ & 5 \\
\hline 180642 & $\ldots$ & 24500 & $3.45 \pm 0.15$ & $12 \pm 3$ & 4 & 24000 & $3.40 \pm 0.15$ & $12 \pm 3$ & 5 \\
\hline 205021 & $\beta$ Cep & 26000 & $3.70 \pm 0.15$ & $6 \pm 3$ & 1 & 25100 & $3.61 \pm 0.15$ & $6 \pm 3$ & 5 \\
\hline 214993 & $12 \mathrm{Lac}$ & 24500 & $3.65 \pm 0.15$ & $10 \pm 4$ & 1 & 23250 & $3.53 \pm 0.15$ & $10 \pm 4$ & 5 \\
\hline
\end{tabular}

Notes: The $1-\sigma$ uncertainty on $T_{\text {eff }}$ is $1000 \mathrm{~K}$ for all stars.

References. (1) Morel et al. 2006; (2) Morel et al. 2008; (3) Hubrig et al. 2008; (4) Morel \& Aerts 2007; (5) this paper.

Table 3. Derived neon abundances.

\begin{tabular}{|c|c|c|c|c|c|c|c|}
\hline \multirow{3}{*}{$\begin{array}{c}\text { HD } \\
\text { Number }\end{array}$} & \multirow{3}{*}{$\begin{array}{l}\text { Alternative } \\
\text { ID }\end{array}$} & \multicolumn{3}{|c|}{ Default $T_{\text {eff }}$ Scale } & \multicolumn{3}{|c|}{ Revised $T_{\text {eff }}$ Scale } \\
\hline & & $\log \epsilon(\mathrm{Ne} \mathrm{I})$ & $\log \epsilon(\mathrm{Ne} I \mathrm{II})$ & $\log \epsilon(\mathrm{Ne})$ & $\log \epsilon(\mathrm{Ne} \mathrm{I})$ & $\log \epsilon(\mathrm{Ne}$ II $)$ & \\
\hline & & $(\operatorname{dex})$ & $(\operatorname{dex})$ & $(\operatorname{dex})$ & & $(\operatorname{dex})$ & \\
\hline 886 & $\gamma$ Peg & $12(12)$ & $7.87 \pm 0.35$ & $8.08 \pm 0.12(13)$ & $.04 \pm 0.12(12)$ & $8.04 \pm 0.35$ & $8.04 \pm 0$ \\
\hline 16582 & & $3(11)$ & $\ldots$ & $13(11)$ & $12(11)$ & $\ldots \quad \ldots$ & $12(11)$ \\
\hline & & $12(8)$ & $7.88 \pm 0.31$ & $8.02 \pm 0.10$ & $12(8)$ & $7.99 \pm 0.31$ & .09 (9) \\
\hline 30836 & $\pi^{4}$ Ori & \pm 0.15 & $\ldots$ & $8.10 \pm 0.15$ & $4 \pm 0.15$ & & $.15(7)$ \\
\hline & & $5(5)$ & $\ldots$ & $15(5)$ & $15(5)$ & $\ldots$ & $15(5)$ \\
\hline 36591 & $\ldots$ & $01 \pm 0.16$ & $7.85 \pm 0.16$ & $7.97 \pm 0.12$ & $16(6)$ & $7.94 \pm 0.16$ & $.10(8)$ \\
\hline 44743 & $\beta \mathrm{CMa}$ & $9 \pm 0.12$ & $7.89 \pm($ & $7.89 \pm 0.06(11)$ & $12(7)$ & $24(4)$ & $11(11)$ \\
\hline 46328 & $\xi^{1} \mathrm{CMa}$ & $01 \pm 0.25$ & $7.91 \pm 0.14$ & $7.96 \pm 0.14(18)$ & $25(10)$ & $7.94 \pm$ & $14(18)$ \\
\hline 50707 & $15 \mathrm{CMa}$ & 21 & $7.73 \pm 0.21$ & $24 \quad(5)$ & $21 \quad(3)$ & $7.96 \pm 0.21$ & $18 \quad(5)$ \\
\hline & $\iota \mathrm{CMa}$ & $14(7)$ & $\ldots \quad \ldots$ & 14 (7) & $14(7)$ & & 14 (7) \\
\hline 520 & $\mathrm{CMa}$ & 14 (6) & $7.74 \pm 0$ & $7.87 \pm 0.11$ & $13(6)$ & $7.86 \pm 0$ & $07 \quad(8)$ \\
\hline & V836 Cen & $8.05 \pm 0.15$ & & $8.03 \pm 0.13$ & $8.01 \pm 0.15$ & $8.01 \pm 0.32$ & 12 (7) \\
\hline 149438 & $\tau$ Sco & $\ldots \quad \ldots$ & $7.98 \pm 0.10$ & $7.98 \pm 0.10$ & $\ldots$ & $7.97 \pm 0.10$ & $10 \quad(5)$ \\
\hline & V2052 Oph & $8.09 \pm 0.11$ & $\ldots \quad \ldots$ & $8.09 \pm 0.11$ & $8.04 \pm 0.11$ & $\ldots \quad \ldots$ & .11 \\
\hline 170580 & $\ldots$ & $8.13 \pm 0.15$ & $\ldots \quad \ldots$ & $8.13 \pm 0.15$ & $8.07 \pm 0.14$ & $\ldots \quad \ldots$ & $8.07 \pm 0.14$ \\
\hline & $\ldots$ & $7.91 \pm 0.18$ & $7.82 \pm 0.27$ & $7.86 \pm 0.16$ & $7.87 \pm 0.18$ & $7.87 \pm 0.27$ & $7.87 \pm 0.15$ \\
\hline 205021 & $\beta$ Cep & $7.99 \pm 0.18$ & $7.82 \pm 0.18$ & $7.94 \pm 0.14$ & $7.93 \pm 0.18$ & $7.93 \pm 0.18$ & $7.93 \pm 0.11$ \\
\hline 214993 & $12 \mathrm{Lac}$ & $8.07 \pm 0.15$ & $7.81 \pm 0.26$ & $8.03 \pm 0.15$ & $7.98 \pm 0.15$ & $7.98 \pm 0.26$ & $7.98 \pm 0.12$ \\
\hline
\end{tabular}

Notes: The abundances are given for both the default (estimated from the Si ionization balance) and the revised $T_{\text {eff }}$ scales (estimated from the $\mathrm{Ne}$ ionization balance for the stars with both Ne I and Ne II abundances, and offset by $-825 \mathrm{~K}$ otherwise; see Sect. 4). In each case we provide the abundances yielded by the Ne I and Ne II lines alone, and the values derived using all lines (on the scale in which $\log \epsilon[\mathrm{H}]=12$ ). The number of lines used for each ion is given in the parentheses. A blank indicates that the spectral features pertaining to a given ionization stage were not measurable.

a mean value: $\log \epsilon(\mathrm{Ne})=8.10 \pm 0.06 \mathrm{dex}^{5}$. The atmospheric parameters were estimated from the NLTE analysis of the $\mathrm{H}$ and Si line profiles, but it should be noted that atmospheric models

\footnotetext{
5 We do not discuss the data for the distant open clusters NGC 6231 , NGC 6611, S285 and S289 (Kilian et al. 1994; Kilian-Montenbruck
}

with an inadequate treatment of metal line blanketing were used (Gold 1984). Considering the similar nature of that sample

et al. 1994) because of the very likely existence of a Galactic Ne abundance gradient (see Sect. 2). 
Table 4. Calculation of the error budget in the case of $\beta \mathrm{CMa}$ (and with the default $T_{\text {eff }}$ scale).

\begin{tabular}{lrrr}
\hline \hline & $\begin{array}{c}\Delta \log \epsilon(\mathrm{Ne} I) \\
(\operatorname{dex})\end{array}$ & $\begin{array}{c}\Delta \log \epsilon(\mathrm{Ne} \text { II }) \\
(\mathrm{dex})\end{array}$ & $\begin{array}{c}\Delta \log \epsilon(\mathrm{Ne}) \\
(\mathrm{dex})\end{array}$ \\
\hline$\sigma_{\text {int }}$ & 0.042 & 0.065 & 0.048 \\
$\sigma_{T_{\text {eff }}}$ & +0.087 & -0.175 & -0.008 \\
$\sigma_{\log g}$ & -0.012 & +0.067 & +0.018 \\
$\sigma_{\xi}$ & -0.004 & -0.012 & -0.008 \\
$\sigma_{T_{\text {eff }} / \log g}$ & +0.068 & -0.117 & +0.001 \\
$\sigma_{\mathrm{T}}$ & 0.119 & 0.231 & 0.053 \\
\hline
\end{tabular}

Notes: $\sigma_{\text {int }}$ : line-to-line scatter; $\sigma_{T_{\text {eff }}}$ : variation of the abundances for $\Delta T_{\text {eff }}=+1000 \mathrm{~K} ; \sigma_{\log g}$ : as before, but for $\Delta \log g=+0.15 \mathrm{dex} ; \sigma_{\xi}$ : as before, but for $\Delta \xi=+3 \mathrm{~km} \mathrm{~s}^{-1} ; \sigma_{T_{\text {eff }} / \log g}$ : as before, but for $\Delta T_{\text {eff }}=$ $+1000 \mathrm{~K}$ and $\Delta \log g=+0.15 \mathrm{dex} ; \sigma_{\mathrm{T}}$ : total uncertainty.

and ours, we can expect the NLTE corrections affecting the Ne II transitions to be of similar magnitude and, as a consequence, to be also small. Assuming $\Delta \epsilon \sim-0.05$ dex (Sect. 4) would lead to a corrected abundance, $\log \epsilon(\mathrm{Ne}) \sim 8.05$ dex, again slightly higher than our best mean estimate. Two stars are in common with our study: HD 36591 and $\tau$ Sco. We find an $\mathrm{Ne}$ abundance lower by 0.11 and $0.18 \mathrm{dex}$, respectively. Even accounting for the small NLTE corrections discussed above, our values appear indeed slightly lower even on a star-to-star basis.

Summarizing, the discussion above shows that the previous determinations in the literature cluster around $\log \epsilon(\mathrm{Ne}) \sim$ $8.1 \mathrm{dex}$ and that our mean abundance lies at the lower end of the proposed values (although marginally compatible within the errors). However, it has to be kept in mind that our study is the first one requiring agreement between two adjacent $\mathrm{Ne}$ ionization stages. Had we only used the Ne I lines, without any constraints imposed by the Ne II lines, we would have inferred a value, $\log \epsilon(\mathrm{Ne})=8.03 \pm 0.08 \mathrm{dex}$, in better agreement with the studies discussed above (Fig. 3; upper, left-hand panel).

\subsection{The Ne content of the Sun from different indicators}

Our mean $\mathrm{Ne}$ abundance is intermediate between the standard solar Ne abundance $(8.08 \pm 0.06 \mathrm{dex}$; GS98) and the most recent estimate following the decrease in the oxygen content $(7.84 \pm$ 0.06 dex; AGS05), and therefore does not support a high neon abundance for the Sun (we note that in principle a more adequate comparison should be made with the Ne abundance at the base of the solar convective zone, which is thought to be some $0.04 \mathrm{dex}$ higher than the surface value because of gravitational element settling; Turcotte et al. 1998).

A reexamination of this quantity was largely motivated by the analysis of highly-ionized Ne IX/X lines in the highresolution spectra of 21 (mostly) strong stellar X-ray emitters collected by the Chandra satellite (Drake \& Testa 2005), which suggested a $[\mathrm{Ne} / \mathrm{O}]$ abundance ratio in the solar corona much higher than the value adopted by AGS05 (taken from Reames 1999): 0.41 vs. $\sim 0.15$. Employing such a high ratio, along with the recently revised solar oxygen abundance $(\log \epsilon[\mathrm{O}] \sim$ 8.66 dex; AGS05), would imply $\log \epsilon(\mathrm{Ne}) \sim 8.27$ dex for the Sun, a value that is potentially high enough to allow the standard solar models to fulfil the helioseismological constraints (8.29 \pm 0.05 dex; Bahcall et al. 2005).

Numerous works have demonstrated a dependence between the abundances of the chemical elements and their first ionization potential (FIP) in stellar transition regions and coronae, with the nature of this relationship depending, however, on the stellar activity level. This phenomenon is still not well understood and a variety of behaviours are observed, but some general trends are emerging (e.g. Audard et al. 2003; Telleschi et al. 2005). First, in relatively quiescent stars (such as the Sun) elements with low FIPs ( $\$ 10 \mathrm{eV}$; e.g. $\mathrm{Mg}, \mathrm{Fe})$ tend to be overabundant compared to the photospheric reference values. In contrast, an opposite trend is observed in strong coronal sources, where these chemical species are depleted with respect to the high-FIP elements (e.g. $\mathrm{N}, \mathrm{O}, \mathrm{Ne}$ ). In view of the significantly different FIPs of $\mathrm{O}$ and $\mathrm{Ne}$ (13.6 and $21.6 \mathrm{eV}$, respectively), a concern is that these elements may suffer various levels of chemical fractionation in the active stars analysed by Drake \& Testa (2005). In moderately active coronal sources or stars in the higher tail of the stellar activity distribution (such as young, fast-rotating dwarfs or some RS CVn binaries in the sample of Drake \& Testa 2005), high $[\mathrm{Ne} / \mathrm{O}]$ abundance ratios are commonly observed (e.g. Brinkman et al. 2001; Wood \& Linsky 2006). As shown by Güdel (2004), this ratio increases in parallel with the characteristic temperature of the plasma in stellar coronae (which is itself correlated with the activity level, as parametrised, for instance, by the ratio of the $\mathrm{X}$-ray to bolometric luminosities). Impulsive, Ne-rich flares are also observed in the Sun, with [Ne/O] enhancements reaching up to a factor $\sim 2$ (Schmelz 1993). A similar situation might prevail in very active stars whose upper atmosphere is believed to be permeated by strong flaring events. Drake \& Testa (2005) addressed this problem and dismissed the possibility that it could affect their conclusions on the basis of the similar $[\mathrm{Ne} / \mathrm{O}] \mathrm{ra}-$ tios obtained for a few stars with low-activity levels. This issue remains controversial, however, as other studies derived much lower ratios in this activity regime (e.g. Liefke \& Schmitt 2006; Raassen et al. 2002). As illustrated by the supplementary data provided by Drake \& Testa (2005), even in the Sun the [Ne/O] measurements in the outer atmosphere show considerable scatter and may depend on the region under investigation (e.g. active regions, flares, wind). Although observations of the quiet Sun clearly favour a low ratio (e.g. Young 2005), definitive statements regarding the value of $[\mathrm{Ne} / \mathrm{O}]$ in the underlying photosphere must probably await a better theoretical understanding of such behaviour. Another limitation affecting the solar $\mathrm{Ne}$ abundance derived from X-ray data is that this quantity is tied to the photospheric oxygen abundance, whose exact value is still being debated (e.g. Antia \& Basu 2006). Taking an element with a well-defined meteoritic abundance as reference (e.g. $\mathrm{Mg}$ ) would avoid this problem, but the abundance ratios of these species relative to Ne are unfortunately more likely to be affected by FIP effects. Taking these two sources of uncertainty together, it might thus prove difficult in the foreseeable future to set very tight constraints on the Ne content of the Sun from coronal observations alone.

Figure 4 shows the Ne abundances recently obtained for different objects in the solar vicinity (excluding peculiar and very evolved objects, such as Wolf-Rayet stars or planetary nebulae). Pre-main sequence stars were also omitted because the Ne abundances generally suffer from large uncertainties and the $[\mathrm{Ne} / \mathrm{O}]$ ratios might be affected by depletion in the X-ray emitting material of oxygen onto dust grains (Drake et al. 2005). This is an unbiased compilation in the sense that this is, to the best of our knowledge, a complete census of the values reported in the literature since Drake \& Testa (2005) study (we include, however, all B-star analyses discussed in Sect. 5.1 and the value for the ionized gas in the Orion nebula derived by Esteban et al. 2004). The oxygen abundance of AGS05 was adopted to obtain the absolute neon abundances from the coronal $[\mathrm{Ne} / \mathrm{O}]$ ratios. For the stars analysed by García-Alvarez et al. (2006, 2008), Ness \& Jordan (2008) and Wood \& Linsky (2006), we used the photospheric 
Table 5. Comparison of the $\log g f$ values used here and those of Seaton (1998).

\begin{tabular}{cccrr}
\hline \hline Lower level & Upper level & $\lambda$ & \multicolumn{2}{c}{$\log g f$} \\
& & $(\AA)$ & This work & Seaton \\
\hline $2 \mathrm{p}^{5}\left({ }^{2} \mathrm{P}_{3 / 2}^{\mathrm{o}}\right) 3 \mathrm{~s}[3 / 2]_{2}$ & $2 \mathrm{p}^{5}\left({ }^{2} \mathrm{P}_{3 / 2}^{\mathrm{o}}\right) 3 \mathrm{p}[3 / 2]_{2}$ & 6143.063 & -0.069 & -0.101 \\
$2 \mathrm{p}^{5}\left({ }^{2} \mathrm{P}_{1 / 2}^{\mathrm{o}}\right) 3 \mathrm{~s}[1 / 2]_{0}$ & $2 \mathrm{p}^{5}\left({ }^{2} \mathrm{P}_{1 / 2}^{\mathrm{o}}\right) 3 \mathrm{p}[1 / 2]_{1}$ & 6163.594 & -0.613 & -0.616 \\
$2 \mathrm{p}^{5}\left({ }^{2} \mathrm{P}_{1 / 2}^{\mathrm{o}}\right) 3 \mathrm{~s}[1 / 2]_{0}$ & $2 \mathrm{p}^{5}\left({ }^{2} \mathrm{P}_{1 / 2}^{\mathrm{o}}\right) 3 \mathrm{p}[3 / 2]_{1}$ & 6266.495 & -0.334 & -0.370 \\
$2 \mathrm{p}^{5}\left({ }^{2} \mathrm{P}_{3 / 2}^{\mathrm{o}}\right) 3 \mathrm{~s}[3 / 2]_{2}$ & $2 \mathrm{p}^{5}\left({ }^{2} \mathrm{P}_{3 / 2}^{\mathrm{o}}\right) 3 \mathrm{p}[5 / 2]_{2}$ & 6334.428 & -0.324 & -0.315 \\
$2 \mathrm{p}^{5}\left({ }^{2} \mathrm{P}_{3 / 2}^{\mathrm{o}}\right) 3 \mathrm{~s}[3 / 2]_{1}$ & $2 \mathrm{p}^{5}\left({ }^{2} \mathrm{P}_{3 / 2}^{\mathrm{o}}\right) 3 \mathrm{p}[3 / 2]_{1}$ & 6382.991 & -0.228 & -0.236 \\
$2 \mathrm{p}^{5}\left({ }^{2} \mathrm{P}_{3 / 2}^{\mathrm{o}}\right) 3 \mathrm{~s}[3 / 2]_{2}$ & $2 \mathrm{p}^{5}\left({ }^{2} \mathrm{P}_{3 / 2}^{\mathrm{o}}\right) 3 \mathrm{p}[5 / 2]_{3}$ & 6402.248 & 0.336 & 0.330 \\
$2 \mathrm{p}^{5}\left({ }^{2} \mathrm{P}_{3 / 2}^{\mathrm{o}}\right) 3 \mathrm{~s}[3 / 2]_{1}$ & $2 \mathrm{p}^{5}\left({ }^{2} \mathrm{P}_{3 / 2}^{\mathrm{o}}\right) 3 \mathrm{p}[5 / 2]_{2}$ & 6506.528 & -0.026 & -0.033 \\
\hline
\end{tabular}

values quoted in these papers. For the metal-poor stars $\xi \mathrm{UMa}$ and Ross 154, we rescaled the solar abundance to the metallicities found by Cayrel de Strobel et al. (1994, [Fe/H] -0.35) and Eggen (1996, [Fe/H] -0.24), respectively. Finally, in the case of the supermetallic binary system $\alpha \mathrm{Cen}([\mathrm{Fe} / \mathrm{H}] \sim+0.24)$, we used the oxygen abundance of Feltzing \& Gonzalez (2001). We caution that the metallicities deviate significantly from solar in these three cases, and that the $\mathrm{Ne}$ abundances may not strictly apply to the Sun. A large scatter is observed in Fig. 4 (total spread of 0.42 dex), but it could be noted that the only few studies supporting a high solar $\mathrm{Ne}$ abundance are generally based on X-ray spectroscopy of strong coronal sources.

\section{Conclusion}

A mean, absolute neon abundance, $\log \epsilon(\mathrm{Ne})=7.97 \pm 0.07 \mathrm{dex}$, has been inferred from our combined NLTE abundance analysis of the photospheric Ne I and Ne II lines in a sample of 18 nearby, early B-type stars. This indicates a value for the Sun $\sim 35 \%$ higher than the new recommended solar abundance $(7.84 \pm$ 0.06 dex; AGS05). In contrast, an increase of the Ne abundance by a factor $\sim 3$ in the solar interior is needed to restore the agreement between the solar models and the helioseismological data. Our results therefore clearly suggest that solving this problem by simply adjusting the solar $\mathrm{Ne}$ abundance would probably require an increase of this quantity well beyond the range of plausible values (note that an enhancement of the other metal abundances to within their uncertainties may also be necessary; Bahcall et al. 2005). We point out that this is a robust conclusion attained regardless of the ion or $T_{\text {eff }}$ scale chosen (Fig. 3).

Neon is produced during carbon burning in the final stages of the evolution of massive stars and one may naturally expect the $\mathrm{Ne}$ abundances of young, B-type stars to be higher than the solar value because of chemical enrichment over the past $4.6 \mathrm{Gyr}$. However, the predicted enhancements in the solar neighbourhood are likely to be very small according to Galactic chemical evolutionary models ( 0.04 dex, Chiappini et al. 2003). Our mean $\mathrm{Ne}$ abundance should therefore be very close to the value prevailing in the protosolar nebula. Unexpectedly, however, metal abundances derived for nearby B stars are often found to be slightly (but significantly) below the most recent estimates for the Sun or the meteoritic values (e.g. Morel 2008, and references therein). This discrepancy may be related to missing physics or unaccounted systematic errors in the B star analyses, and may question the assumption that the neon abundance derived from hot stars is directly transposable to the Sun. The fact that our mean $\mathrm{Ne}$ abundance is indistinguishable from the values recently determined for the ionized gas in the Orion nebula $(8.05 \pm 0.07$ dex, Esteban et al. 2004), within supergranules $(7.89 \pm 0.13 \mathrm{dex}$, Young 2005) or from in situ observations of the

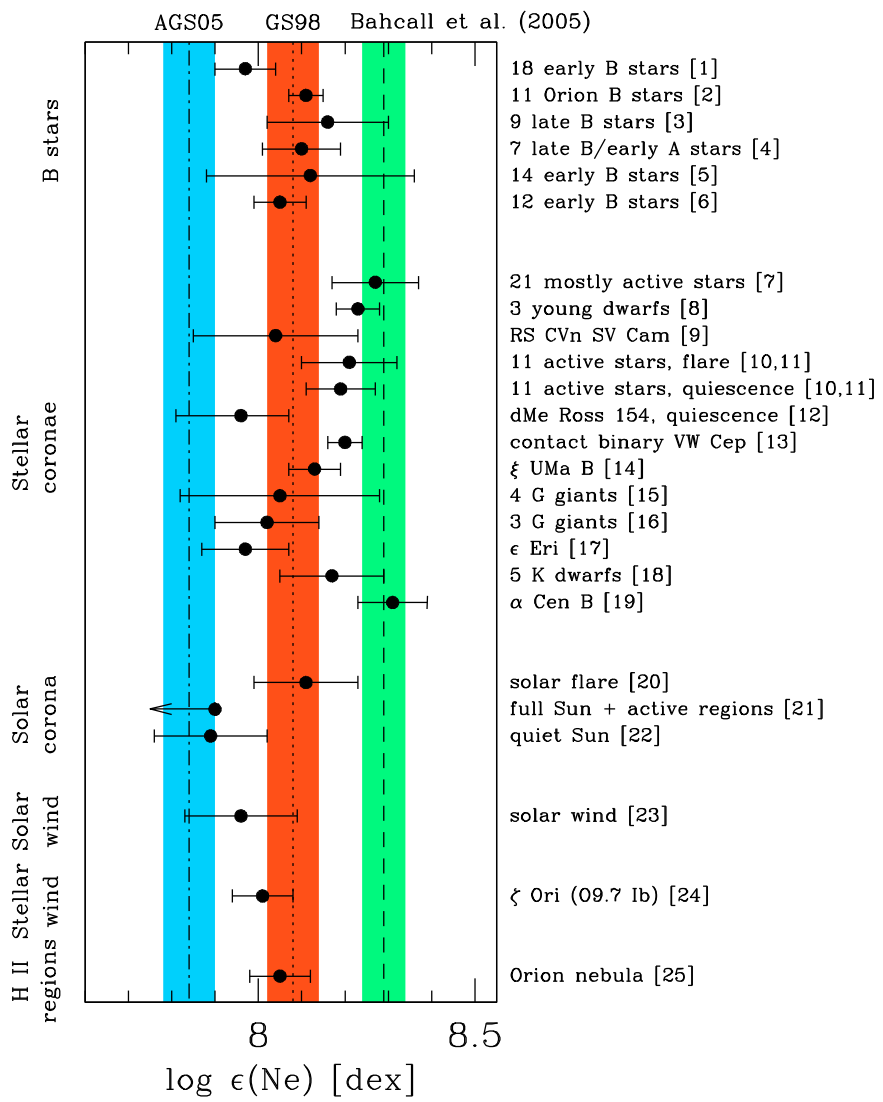

Fig. 4. Neon abundances from different indicators recently reported in the literature. The stellar coronal sources are roughly ordered as a function of decreasing activity from top to bottom. The long-dashed line indicates the $\mathrm{Ne}$ abundance needed to reconcile the solar interior models and the results of helioseismology $(8.29 \pm 0.05 \mathrm{dex}$, Bahcall et al. 2005), while the dotted and dashed-dotted lines show the standard (8.08 \pm 0.06 dex, GS98) and the new (7.84 \pm 0.06 dex, AGS05) solar Ne abundances, respectively. The vertical, shaded strips show the corresponding uncertainties. In the online version of this journal, these are plotted in green, red and blue, respectively. References: (1) this paper; (2) Cunha et al. (2006); (3) Hempel \& Holweger (2003); (4) Dworetsky \& Budaj (2000); (5) Sigut (1999); (6) Kilian (1994), but corrected by -0.05 dex (see text); (7) Drake \& Testa (2005); (8) García-Alvarez et al. (2008); (9) Sanz-Forcada et al. (2006); (10) Nordon \& Behar (2007); (11) Nordon \& Behar (2008); (12) Wargelin et al. (2008); (13) Huenemoerder et al. (2006); (14) Ball et al. (2005); (15) Ayres et al. (2007); (16) García-Alvarez et al. (2006); (17) Ness \& Jordan (2008); (18) Wood \& Linsky (2006); (19) Liefke \& Schmitt (2006); (20) Landi et al. (2007); (21) Schmelz et al. (2005) (upper limit); (22) Young (2005); (23) Bochsler (2007); (24) Raassen et al. (2008); (25) Esteban et al. (2004). In the case of (8) and (16), we used the abundances derived from differential emission measure (DEM) reconstruction. 
solar wind (7.96 \pm 0.13 dex, Bochsler 2007) nevertheless suggests that the abundances we derived are representative of the solar value (see Fig. 4).

Acknowledgements. Thierry Morel acknowledges financial support from the Research Council of Leuven University through grant GOA/2003/04 and Belspo for contract PRODEX GAIA-DPAC. We are grateful to Nicolas Grevesse for interesting discussions about the neon abundance of the Sun and useful comments on the first draft of this paper. It is also a pleasure to thank Giusi Micela for precisions about coronal abundances. The careful reading of the manuscript and the useful suggestions from the anonymous referee were greatly appreciated. We are indebted to all the observers who contributed to the acquisition of the data used in this paper. The archival ELODIE data have been processed within the PLEINPOT environment.This research made use of NASA's Astrophysics Data System Bibliographic Services, the SIMBAD database operated at CDS, Strasbourg (France).

\section{References}

Aerts, C., De Cat, P., Handler, G., et al. 2004, MNRAS, 347, 463

Aller, L. H., \& Jugaku, J. 1958, ApJ, 127, 125

Antia, H. M., \& Basu, S. 2006, ApJ, 644, 1292

Asplund, M., Grevesse, N., \& Sauval, A. J. 2005, in Cosmic abundances as records of stellar evolution and nucleosynthesis, ed. T. G. Barnes III, \& F. N. Bash, ASP Conf. Ser., 336, 25 (AGS05)

Audard, M., Güdel, M., Sres, A., Raassen, A. J. J., \& Mewe, R. 2003, A\&A, 398, 1137

Auer, L. H., \& Mihalas, D. 1973, ApJ, 184, 151

Ayres, T. R., Hodges-Kluck, E., \& Brown, A. 2007, ApJS, 171, 304

Bahcall, J. N., Basu, S., \& Serenelli, A. M. 2005, ApJ, 631, 1281

Ball, B., Drake, J. J., Lin, L., et al. 2005, ApJ, 634, 1336

Basu, S., \& Antia, H. M. 2008, Phys. Rep., 457, 217

Basu, S., Chaplin, W. J., Elsworth, Y., et al. 2007, ApJ, 655, 660

Bochsler, P. 2007, A\&A, 471, 315

Brinkman, A. C., Behar, E., Güdel, M., et al. 2001, A\&A, 365, L324

Butler, K. 2008a, A\&A, submitted

Butler, K. 2008b, A\&A, submitted

Butler, K. 2008c, A\&A, submitted

Butler, K. 2008d, A\&A, submitted

Cayrel de Strobel, G., Cayrel, R., Friel, E., Zahn, J.-P., \& Bentolila, C. 1994, A\&A, 291, 505

Chiappini, C., Romano, D., \& Matteucci, F. 2003, MNRAS, 339, 63

Cowley, C. R. 1971, Observatory, 91, 139

Cunha, K., \& Lambert, D. L. 1992, A\&A, 399, 586

Cunha, K., Hubeny, I., \& Lanz, T. 2006, ApJ, 647, L143

Delahaye, F., \& Pinsonneault, M. H. 2006, ApJ, 649, 529

Drake, J. J., \& Testa, P. 2005, Nature, 436, 525

Drake, J. J., Brickhouse, N. S., Kashyap, V. L., et al. 2001, ApJ, 548, L81

Drake, J. J., Testa, P., \& Hartmann, L. 2005, ApJ, 627, L149

Dworetsky, M. M., \& Budaj, J. 2000, MNRAS, 318, 1264

Eggen, O. J. 1996, AJ, 111, 466

Esteban, C., Peimbert, M., García-Rojas, J., et al. 2004, MNRAS, 355, 229

Feltzing, S., \& Gonzalez, G. 2001, A\&A, 367, 253

García-Alvarez, D., Drake, J. J., Ball, B., Lin, L., \& Kashyap, V. L. 2006, ApJ, 638, 1028

García-Alvarez, D., Drake, J. J., Kashyap, V. L., Lin, L., \& Ball, B. 2008, ApJ, 679,1509

Gies, D. R., \& Lambert, D. L. 1992, ApJ, 387, 673

Gold, M. 1984, Diplomarbeit, Ludwig Maximilian Universität, München, Germany

Grevesse, N., \& Sauval, A. J. 1998, Space Sci. Rev., 85, 161 (GS98)

Griffin, D. C., Mitnik, D. M., \& Badnell, N. R. 2001, J. Phys. B, 34, 4401

Güdel, M. 2004, A\&AR, 12, 71

Hempel, M., \& Holweger, H. 2003, A\&A, 408, 1065

Henry, R. B. C., Kwitter, K. B., \& Balick, B. 2004, AJ, 127, 2284

Hubeny, I., \& Lanz, T. 1995, ApJ, 439, 875

Hubrig, S., Briquet, M., Morel, T., et al. 2008, A\&A, in press
Huenemoerder, D. P., Testa, P., \& Buzasi, D. L. 2006, ApJ, 650, 1119 Hummer, D. G., Berrington, K. A., Eissner, W., et al. 1993, A\&A, 279, 298 Kilian, J. 1994, A\&A, 282, 867

Kilian, J., \& Nissen, P. E. 1989, A\&AS, 80, 255

Kilian, J., Montenbruck, O., \& Nissen, P. E. 1994, A\&A, 284, 437

Kilian-Montenbruck, J., Gehren, T., \& Nissen, P. E. 1994, A\&A, 291, 757

Kramida, A. E., \& Nave, G. 2006a, Eur. Phys. J. D, 39, 331

Kramida, A. E., \& Nave, G. 2006b, Eur. Phys. J. D, 37, 1

Kramida, A. E., Brown, C. M., Feldman, U., \& Reader, J. 2006, Phys. Scr., 74, 156

Kurucz, R. L. 1993, ATLAS9 Stellar Atmosphere Programs and 2 km/s grid, Kurucz CD-ROM No. 13, Cambridge, Mass.: Smithsonian Astrophysical Observatory

Kurucz, R. L., \& Bell, B. 1995, Atomic Line Data, ed. R. L. Kurucz, \& B. Bell, Kurucz CD-ROM No. 23, Cambridge, Mass.: Smithsonian Astrophysical Observatory

Landi, E., Feldman, U., \& Doschek, G. A. 2007, ApJ, 659, 743

Liefke, C., \& Schmitt, J. H. M. M. 2006, A\&A, 458, L1

Luyten, W. J. 1936, ApJ, 84, 85

Lyubimkov, L. S., Rachkovskaya, T. M., Rostopchin, S. I., \& Lambert, D. L. 2002, MNRAS, 333, 9

Martin, J. C. 2004, AJ, 128, 2474

Martín-Hernández, N. L., Peeters, E., Morisset, C., et al. 2002, A\&A, 381, 606

Mazumdar, A., Briquet, M., Desmet, M., \& Aerts, C. 2006, A\&A, 459, 589

Morel, T. 2008, in Evolution and Pulsation of Massive Stars on the Main Sequence and Close to it, CoAst, submitted

Morel, T., \& Aerts, C. 2007, CoAst, 150, 201

Morel, T., \& Butler, K. 2007, in Unsolved Problems in Stellar Physics, AIP Conf. Proc. Ser., 948, 225

Morel, T., \& Butler, K. 2008, in Helioseismology, Asteroseismology and MHD Connections, J. Phys. Conf. Ser., in press

Morel, T., Butler, K., Aerts, C., Neiner, C., \& Briquet, M. 2006, A\&A, 457, 651

Morel, T., Hubrig, S., \& Briquet, M. 2008, A\&A, 481, 453

Neiner, C., Henrichs, H. F., Floquet, M., et al. 2003, A\&A, 411, 565

Ness, J.-U., \& Jordan, C. 2008, MNRAS, 385, 1691

Nieva, M. F., \& Przybilla, N. 2007, A\&A, 467, 295

Nordon, R., \& Behar, E. 2007, A\&A, 464, 309

Nordon, R., \& Behar, E. 2008, A\&A, 482, 639

Raassen, A. J. J., Mewe, R., \& Audard, M. 2002, A\&A, 389, 228

Raassen, A. J. J., van der Hucht, K. A., Miller, N. A., \& Cassinelli, J. P. 2008, A\&A, 478, 513

Ralchenko, Yu., Kramida, A. E., Reader, J., and NIST ASD Team 2008, NIST Atomic Spectra Database, version 3.1.4, National Institute of Standards and Technology, Gaithersburg, MD, http://physics.nist.gov/asd3

Reames, D. V. 1999, Space Sci. Rev., 90, 413

Roberts, L. C., Jr., Turner, N. H., \& Ten Brummelaar, T. A. 2007, AJ, 133, 545

Saloman, E. B., \& Sansonetti, C. J. 2004, J. Phys. Chem. Ref. Data, 33, 1113

Sanz-Forcada, J., Favata, F., \& Micela, G. 2006, A\&A, 445, 673

Schmelz, J. T. 1993, ApJ, 408, 373

Schmelz, J. T., Nasraoui, K., Roames, J. K., Lippner, L. A., \& Garst, J. W. 2005, ApJ, 634, L197

Schnerr, R. S., Henrichs, H. F., Oudmaijer, R. D., \& Telting, J. H. 2006, A\&A, 459, L21

Seaton, M. J. 1962, in Atomic and Molecular Processes, ed. D. R. Bates (New York: Academic Press)

Seaton, M. J. 1998, J. Phys. B, 31, 5315

Sigut, T. A. A. 1999, ApJ, 519, 303

Stankov, A., \& Handler, G. 2005, ApJS, 158, 193

Telleschi, A., Güdel, M., Briggs, K., et al. 2005, ApJ, 622, 653

Turcotte, S., Richer, J., Michaud, G., Iglesias, C. A., \& Rogers, F. J. 1998, ApJ, 504,539

Van Regemorter, H. 1962, ApJ, 136, 906

Wargelin, B. J., Kashyap, V. L., Drake, J. J., García-Alvarez, D., \& Ratzlaff, P. W. 2008, ApJ, 676, 610

Wood, B. E., \& Linsky, J. L. 2006, ApJ, 643, 444

Young, P. R. 2005, A\&A, 444, L45

Zaatri, A., Provost, J., Berthomieu, G., Morel, P., \& Corbard, T. 2007, A\&A, 469, 1145 
T. Morel and K. Butler: The neon content of nearby B-type stars, Online Material $p 1$

\section{Appendix A: Examples of comparison between \\ observed and synthetic $\mathrm{Ne}$ line profiles}

Figures A.1-A.4 show comparisons between the observed and the synthetic Ne line profiles for four representative stars sampling the whole $T_{\text {eff }}$ range and with different data quality: $\iota \mathrm{CMa}$, $\gamma$ Peg, $\xi^{1}$ CMa and $\tau$ Sco. 
T. Morel and K. Butler: The neon content of nearby B-type stars, Online Material $p 2$

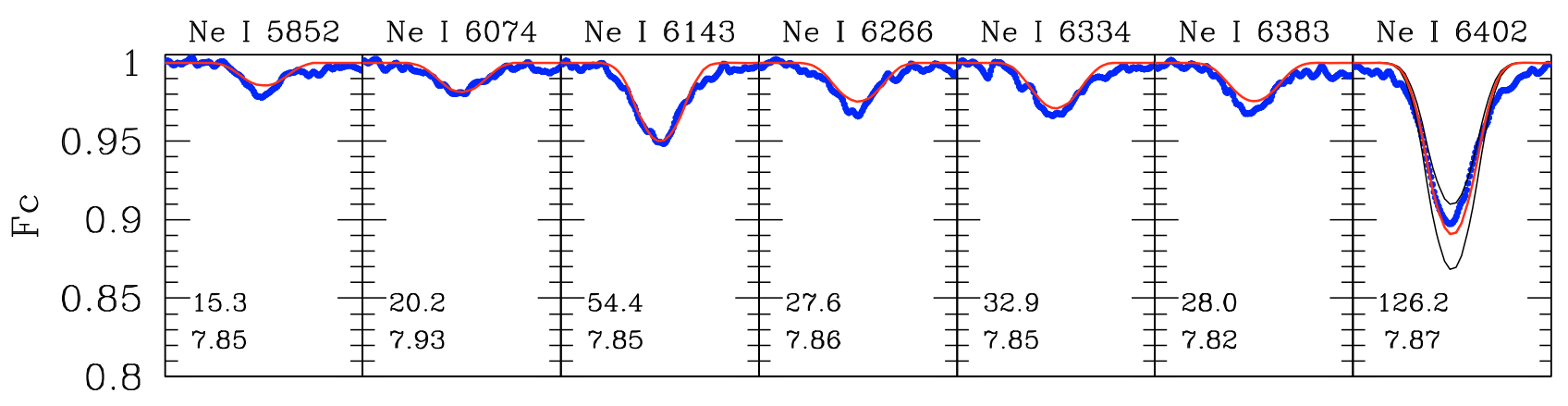

Wavelength $( \pm 2 \AA)$

Fig. A.1. Comparison for $\iota$ CMa between the observed (blue dots) and the synthetic profiles (red line) computed for the abundance given by the corresponding spectral line (value given in every panel, along with the measured $E W$; see Table B.1). The synthetic spectra were convolved with a rotational broadening function with $v \sin i=32 \mathrm{~km} \mathrm{~s}^{-1}$ (Morel et al. 2008). The effect of varying the abundance by \pm 0.1 dex is shown for Ne I 16402 . The spectral range in each panel is $4 \AA$.
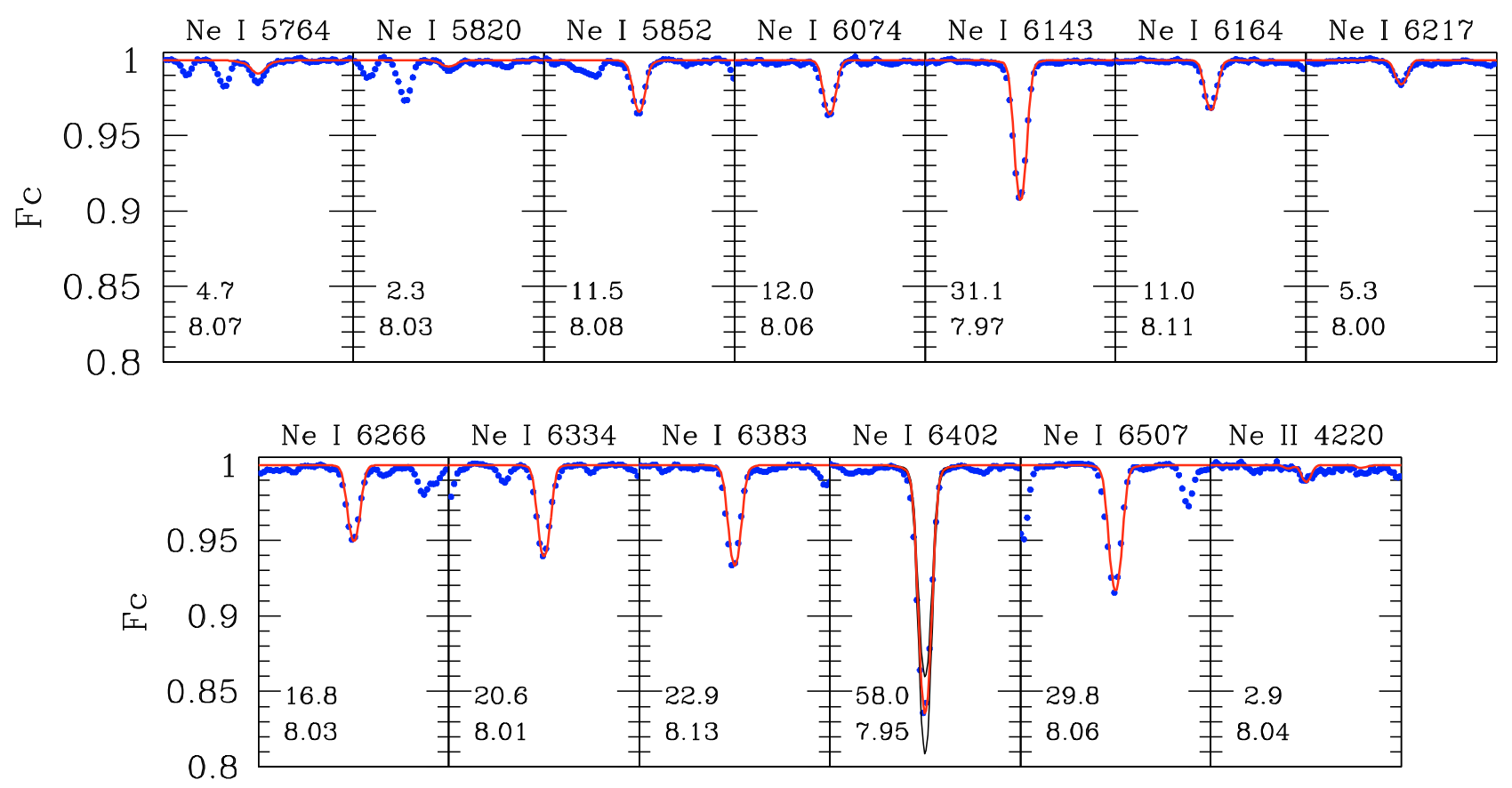

Wavelength $( \pm 2 \AA)$

Fig. A.2. Same as Fig. A.1, but for $\gamma$ Peg. The synthetic spectra were convolved with a rotational broadening function with $v \sin i=10 \mathrm{~km} \mathrm{~s}^{-1}$ (Morel et al. 2008). 
T. Morel and K. Butler: The neon content of nearby B-type stars, Online Material $p 3$
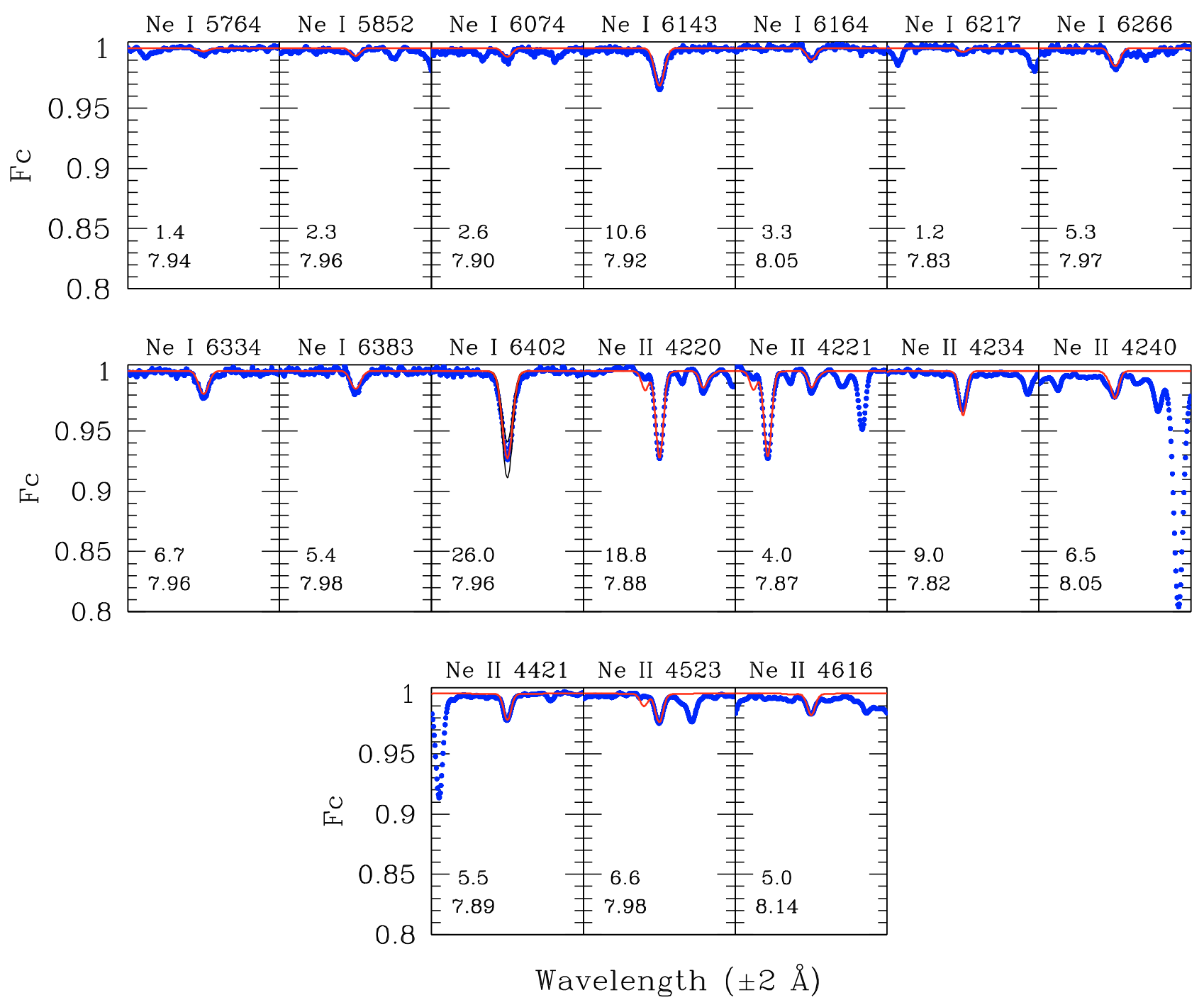

Fig. A.3. Same as Fig. A.1, but for $\xi^{1}$ CMa. The synthetic spectra were convolved with a rotational broadening function with $v \sin i=10 \mathrm{~km} \mathrm{~s}^{-1}$ (Morel et al. 2008). Ne II $\lambda 4392$ has been omitted in this figure, as it falls in the wing of He I $\lambda 4387.9$ (which is not included in the model calculations).

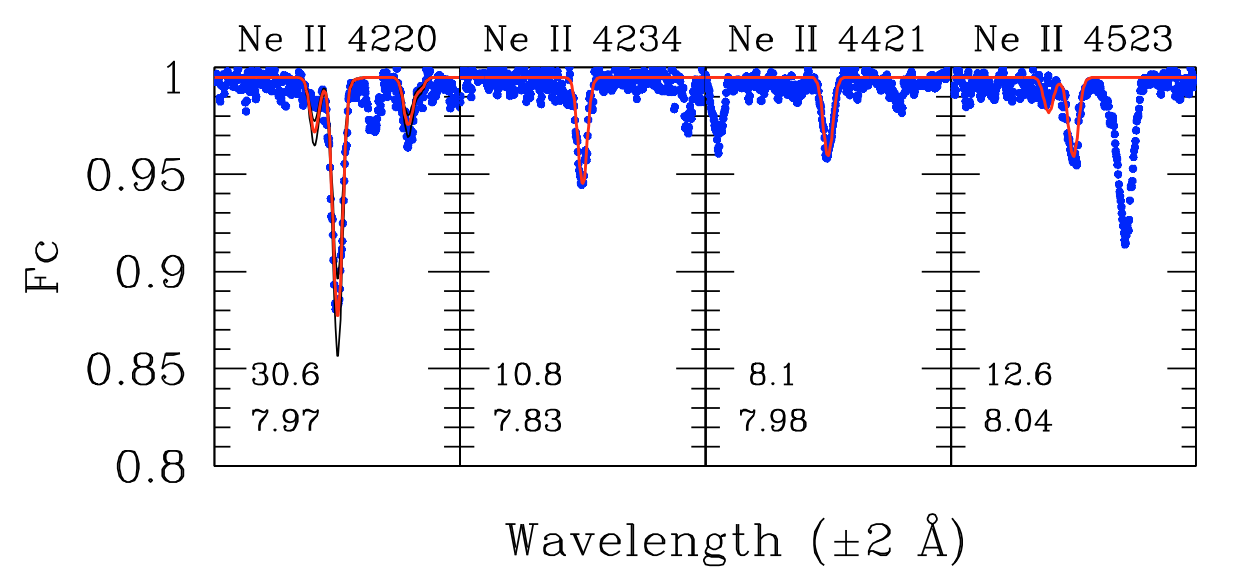

Fig. A.4. Same as Fig. A.1, but for $\tau$ Sco. The synthetic spectra were convolved with a rotational broadening function with $v \sin i=8 \mathrm{~km} \mathrm{~s}{ }^{-1}$ (Hubrig et al. 2008). The effect of varying the abundance by \pm 0.1 dex is shown for Ne II $\lambda 4220$. Ne II $\lambda 4392$ has been omitted in this figure, as it falls in the wing of He I $\lambda 4387.9$ (which is not included in the model calculations). 
T. Morel and K. Butler: The neon content of nearby B-type stars, Online Material p 4

\section{Appendix B: log gf values, EW measurements}

and line-by-line abundances

Table B.1 lists the adopted $\log g f$ values, measured $E W$ s and individual abundances for the neon lines used in the analysis. 
T. Morel and K. Butler: The neon content of nearby B-type stars, Online Material p 5

Table B.1. Adopted $\log g f$ values, $E W$ measurements (in $\mathrm{m} \AA$ ) and line-by-line abundances (on the scale in which $\log \epsilon[\mathrm{H}]=12$ ).

\begin{tabular}{|c|c|c|c|c|c|c|c|c|c|c|c|c|c|c|c|c|c|c|c|}
\hline \multirow{2}{*}{$\begin{array}{l}\text { Transition }^{a} \\
\mathbf{N e} \mathbf{I}\end{array}$} & \multirow[t]{2}{*}{$\log g f$} & \multicolumn{2}{|c|}{$\begin{array}{c}\text { HD } 886 \\
\gamma \text { Peg }\end{array}$} & \multicolumn{2}{|c|}{$\begin{array}{c}\text { HD } 16582 \\
\delta \text { Cet }\end{array}$} & \multicolumn{2}{|c|}{$\begin{array}{c}\text { HD } 29248 \\
v \text { Eri }\end{array}$} & \multicolumn{2}{|c|}{$\begin{array}{c}\text { HD } 30836 \\
\pi^{4} \text { Ori }\end{array}$} & \multicolumn{2}{|c|}{$\begin{array}{c}\text { HD } 35468 \\
\gamma \text { Ori }\end{array}$} & \multicolumn{2}{|c|}{ HD 36591} & \multicolumn{2}{|c|}{$\begin{array}{c}\mathrm{HD} 44743 \\
\beta \mathrm{CMa}\end{array}$} & \multicolumn{2}{|c|}{$\begin{array}{c}\mathrm{HD} 46328 \\
\xi^{1} \mathrm{CMa}\end{array}$} & \multicolumn{2}{|c|}{$\begin{array}{c}\text { HD } 50707 \\
15 \text { CMa }\end{array}$} \\
\hline & & & & & & & & & & & & & & & & & & & \\
\hline 5764.42 & -0.316 & 4.7 & 8.07 & 2.9 & 7.89 & 3.2 & 7.98 & $\ldots$ & $\ldots$ & $\ldots$ & $\ldots$ & $\ldots$ & $\ldots$ & 2.0 & 7.83 & 1.4 & 7.94 & $\ldots$ & $\ldots$ \\
\hline 5820.16 & -0.517 & 2.3 & 8.03 & $\ldots$ & & $\ldots$ & $\ldots$ & $\ldots$ & 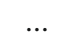 & $\ldots$ & $\ldots$ & $\ldots$ & $\ldots$ & $\ldots$ & $\ldots$ & & & $\ldots$ & $\ldots$ \\
\hline 5852.49 & -0.453 & 11.5 & 8.08 & 10.1 & 8.06 & $\ldots$ & $\ldots$ & 13.3 & 8.08 & $\ldots$ & $\ldots$ & $\ldots$ & $\ldots$ & $\ldots$ & $\ldots$ & 2.3 & 7.96 & $\ldots$ & $\ldots$ \\
\hline 6074.34 & -0.508 & 12.0 & 8.06 & 10.0 & 8.01 & $\ldots$ & $\ldots$ & $\ldots$ & $\ldots$ & $\ldots$ & $\ldots$ & 4.7 & 7.96 & 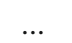 & $\ldots$ & 2.6 & 7.90 & & \\
\hline 6143.06 & -0.069 & 31.1 & 7.97 & 27.3 & 7.94 & 28.1 & 7.96 & 33.1 & 7.92 & 32.6 & 7.91 & 14.2 & 7.88 & 18.0 & 7.85 & 10.6 & 7.92 & 19.7 & 7.92 \\
\hline 6163.59 & -0.613 & 11.0 & 8.11 & 9.2 & 8.06 & 8.2 & 8.04 & 10.6 & 8.01 & $\ldots$ & $\ldots$ & $\ldots$ & $\ldots$ & 5.0 & 7.92 & 3.3 & 8.05 & $\ldots$ & $\ldots$ \\
\hline 6217.28 & -0.936 & 5.3 & 8.00 & 4.2 & 7.93 & $\ldots$ & $\ldots$ & $\ldots$ & $\ldots$ & $\ldots$ & $\ldots$ & ( & $\ldots$ & $\ldots$ & $\ldots$ & 1.2 & 7.83 & $\ldots$ & $\ldots$ \\
\hline 6266.49 & -0.334 & 16.8 & 8.03 & 15.2 & 8.01 & 16.1 & 8.06 & 24.6 & 8.11 & 18.9 & 8.00 & 6.7 & 7.91 & $\ldots$ & $\ldots$ & 5.3 & 7.97 & $\ldots$ & $\ldots$ \\
\hline 6334.43 & -0.324 & 20.6 & 8.01 & 19.8 & 8.03 & 18.3 & 8.00 & 26.3 & 8.05 & 27.8 & 8.08 & $\ldots$ & $\ldots$ & 11.1 & 7.88 & 6.7 & 7.96 & $\ldots$ & $\ldots$ \\
\hline 6382 & -0.228 & 22.9 & 8.13 & 19.7 & 8.09 & 16.4 & 8.03 & 30.6 & 8.18 & 24.5 & 8.08 & 9.7 & 8.04 & 9.9 & 7.92 & 5.4 & 7.98 & 13.9 & 8.10 \\
\hline 25 & 0.336 & 58.0 & 7.95 & 55.3 & 7.96 & 57.1 & 7.92 & 72.7 & 7.93 & 65.0 & 7.85 & 31.5 & 7.89 & 41.7 & 7.86 & 26.0 & 7.96 & 38.3 & 7.87 \\
\hline 6506.53 & -0.026 & 29.8 & 8.06 & 22.6 & 7.96 & 23.1 & 7.98 & $\ldots$ & $\ldots$ & $\ldots$ & $\ldots$ & 12.3 & 7.95 & 16.3 & 7.94 & $\ldots$ & $\ldots$ & $\ldots$ & $\ldots$ \\
\hline \multicolumn{20}{|c|}{0.0022 .01 .7025 .1} \\
\hline 4219.74 & 0.674 & 2.9 & 8.04 & $\ldots$ & $\ldots$ & 6.2 & 7.99 & $\ldots$ & $\ldots$ & $\ldots$ & $\ldots$ & 14.1 & 7.94 & 10.2 & 7.84 & 18.8 & 7.88 & 16.3 & 8.05 \\
\hline 9 & -0.179 & $\ldots$ & $\ldots$ & $\ldots$ & $\ldots$ & $\ldots$ & $\ldots$ & $\ldots$ & $\ldots$ & $\ldots$ & $\ldots$ & $\ldots$ & $\ldots$ & $\ldots$ & $\ldots$ & 4.0 & 7.87 & $\ldots$ & $\ldots$ \\
\hline 5 & 0.362 & $\ldots$ & $\ldots$ & $\ldots$ & $\ldots$ & $\ldots$ & $\ldots$ & $\ldots$ & $\ldots$ & $\ldots$ & $\ldots$ & 7.0 & 7.95 & $\ldots$ & $\ldots$ & 9.0 & 7.82 & $\ldots$ & $\ldots$ \\
\hline 4240 & -0.089 & $\ldots$ & $\ldots$ & $\ldots$ & $\ldots$ & $\ldots$ & $\ldots$ & $\ldots$ & $\ldots$ & $\ldots$ & $\ldots$ & $\ldots$ & $\ldots$ & $\ldots$ & $\ldots$ & 6.5 & 8.05 & $\ldots$ & $\ldots$ \\
\hline $4391.99^{b}$ & 0.908 & $\ldots$ & $\ldots$ & $\ldots$ & $\ldots$ & $\ldots$ & $\ldots$ & $\ldots$ & $\ldots$ & $\ldots$ & $\ldots$ & $\ldots$ & $\ldots$ & 13.4 & 7.93 & 25.5 & 7.93 & 12.2 & 7.87 \\
\hline 4421.39 & 0.158 & $\ldots$ & $\ldots$ & $\ldots$ & $\ldots$ & $\ldots$ & $\ldots$ & $\ldots$ & $\ldots$ & $\ldots$ & $\ldots$ & $\ldots$ & $\ldots$ & 2.2 & 7.82 & 5.5 & 7.89 & $\ldots$ & $\ldots$ \\
\hline & 0.154 & $\ldots$ & $\ldots$ & $\ldots$ & $\ldots$ & $\ldots$ & $\ldots$ & $\ldots$ & $\ldots$ & $\ldots$ & $\ldots$ & $\ldots$ & $\ldots$ & 3.8 & 7.95 & 6.6 & 7.98 & $\ldots$ & $\ldots$ \\
\hline 4616.09 & -0.117 & $\ldots$ & $\ldots$ & $\ldots$ & $\ldots$ & $\ldots$ & $\ldots$ & $\ldots$ & $\ldots$ & $\ldots$ & $\ldots$ & $\ldots$ & $\ldots$ & $\ldots$ & $\ldots$ & 5.0 & 8.14 & $\ldots$ & $\ldots$ \\
\hline Transition $^{a}$ & $\log g f$ & \multicolumn{2}{|c|}{$\begin{array}{c}\text { HD } 51309 \\
\iota \text { CMa }\end{array}$} & \multicolumn{2}{|c|}{$\begin{array}{c}\text { HD } 52089 \\
\epsilon \text { CMa }\end{array}$} & $\begin{array}{r}\text { HD } 1 \\
\text { V83 }\end{array}$ & $\begin{array}{l}29929 \\
6 \text { Cen }\end{array}$ & HD 1 & o & $\begin{array}{l}\text { HD } \\
\text { V20 }\end{array}$ & & & 30 & HD & & $\begin{array}{r}\mathrm{HD} \\
\beta\end{array}$ & $\mathrm{p}$ & $\begin{array}{r}\mathrm{HD} \\
12\end{array}$ & \\
\hline $\mathrm{Ne} I$ & & & & & & & & & & & & & & & & & & & \\
\hline & -0.316 & $\ldots$ & $\ldots$ & $\ldots$ & $\ldots$ & $\ldots$ & $\ldots$ & $\ldots$ & $\ldots$ & $\ldots$ & $\ldots$ & 7.7 & 8.19 & $\ldots$ & $\ldots$ & $\ldots$ & $\ldots$ & $\ldots$ & $\ldots$ \\
\hline 5820 & -0.517 & $\ldots$ & $\ldots$ & $\ldots$ & $\ldots$ & $\ldots$ & $\ldots$ & $\ldots$ & $\ldots$ & $\ldots$ & $\ldots$ & $\ldots$ & $\ldots$ & $\ldots$ & $\ldots$ & $\ldots$ & $\ldots$ & $\ldots$ & $\ldots$ \\
\hline & -0.453 & 15.3 & 7.85 & $\ldots$ & $\ldots$ & $\ldots$ & & - & - & $\cdots$ & $\ldots$ & 19.7 & 8.11 & $\cdots$ & $\ldots$ & $\ldots$ & $\ldots$ & $\ldots$ & 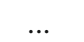 \\
\hline 60 & -0.508 & 20.2 & 7.93 & $\ldots$ & & $\ldots$ & & ( & 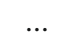 & $\ldots$ & $\ldots$ & 23.2 & 8.15 & & $\ldots$ & $\ldots$ & $\ldots$ & 11.0 & 8.14 \\
\hline 61 & -0.069 & 54.4 & 7.85 & 21.6 & 7.83 & 23.1 & 7.94 & & & 34.1 & 8.05 & 44.3 & 7.97 & 23.6 & 7.99 & 14.8 & 7.86 & & \\
\hline 6163.59 & -0.613 & $\ldots$ & $\ldots$ & $\ldots$ & $\ldots$ & $\ldots$ & $\ldots$ & $\ldots$ & 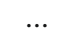 & $\ldots$ & $\ldots$ & 19.2 & 8.18 & $\ldots$ & $\ldots$ & $\ldots$ & $\ldots$ & 7.7 & 8.06 \\
\hline 17.28 & -0.936 & $\ldots$ & $\ldots$ & $\ldots$ & $\ldots$ & $\ldots$ & 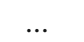 & $\ldots$ & 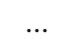 & $\ldots$ & $\ldots$ & 11.3 & 8.14 & $\ldots$ & $\ldots$ & $\ldots$ & $\ldots$ & $\ldots$ & \\
\hline 66.49 & -0.334 & 27.6 & 7.86 & 14.0 & 7.97 & 14.6 & 8.09 & $\ldots$ & $\ldots$ & $\ldots$ & $\ldots$ & 23.2 & 7.99 & $\ldots$ & $\ldots$ & $\ldots$ & $\ldots$ & 10.9 & 7.93 \\
\hline 34.43 & -0.324 & 32.9 & 7.85 & 12.6 & 7.83 & 21.0 & 8.14 & $\ldots$ & $\ldots$ & $\ldots$ & $\ldots$ & 33.2 & 8.05 & $\ldots$ & $\ldots$ & 12.6 & 8.03 & 15.0 & 7.96 \\
\hline 6382.99 & -0.228 & 28.0 & 7.82 & 11.7 & 7.88 & 14.1 & 8.03 & $\ldots$ & $\ldots$ & $\ldots$ & $\ldots$ & 32.9 & 8.09 & $\ldots$ & $\ldots$ & 10.6 & 8.05 & $\ldots$ & $\ldots$ \\
\hline 6402.25 & 0.336 & 126.2 & 7.87 & 48.6 & 7.82 & 42.5 & 7.86 & $\ldots$ & $\ldots$ & 65.6 & 8.04 & 73.8 & 7.89 & 41.6 & 7.88 & 30.9 & 7.83 & 50.6 & 7.90 \\
\hline 6506.53 & -0.026 & $\ldots$ & $\ldots$ & 16.8 & 7.84 & 21.0 & 8.01 & $\ldots$ & $\ldots$ & $\ldots$ & $\ldots$ & 44.5 & 8.05 & 9.9 & 7.74 & 11.4 & 7.88 & 17.6 & 7.91 \\
\hline & & & & & & & & & & & & & & & & & & & \\
\hline & 0.674 & $\cdots$ & $\ldots$ & 7.2 & 7.86 & $\ldots$ & $\ldots$ & 30.6 & 7.97 & $\ldots$ & $\ldots$ & $\cdots$ & $\ldots$ & 16.5 & 8.02 & 15.3 & 7.95 & 9.4 & 7.98 \\
\hline & -0.179 & $\ldots$ & $\ldots$ & $\ldots$ & $\ldots$ & $\ldots$ & $\ldots$ & $\ldots$ & $\ldots$ & $\ldots$ & $\ldots$ & $\ldots$ & $\ldots$ & $\ldots$ & $\cdots$ & $\ldots$ & $\ldots$ & $\ldots$ & $\ldots$ \\
\hline & 0.362 & ... & $\ldots$ & ... & $\ldots$ & ... & $\ldots$ & 10.8 & 7.83 & ... & ... & $\cdots$ & ... & 5.6 & 7.87 & ... & ... & $\ldots$ & $\ldots$ \\
\hline & -0.089 & $\ldots$ & $\ldots$ & $\ldots$ & $\ldots$ & $\ldots$ & $\ldots$ & $\ldots$ & $\ldots$ & $\ldots$ & $\ldots$ & $\ldots$ & $\ldots$ & $\ldots$ & $\ldots$ & $\ldots$ & $\ldots$ & $\ldots$ & $\ldots$ \\
\hline & 0.908 & $\ldots$ & $\ldots$ & 8.0 & 7.86 & 7.9 & 8.01 & 35.3 & 8.05 & & & $\cdots$ & $\ldots$ & 10.0 & 7.72 & 14.5 & 7.91 & $\ldots$ & $\ldots$ \\
\hline 4 & 0.158 & & $\ldots$ & $\ldots$ & $\ldots$ & $\ldots$ & $\ldots$ & 8.1 & 7.98 & $\cdots$ & $\cdots$ & $\cdots$ & $\ldots$ & $\ldots$ & $\ldots$ & $\ldots$ & $\ldots$ & $\ldots$ & $\ldots$ \\
\hline & 0.154 & $\ldots$ & $\ldots$ & $\ldots$ & $\ldots$ & $\ldots$ & $\cdots$ & 12.6 & 8.04 & & & $\ldots$ & $\ldots$ & $\ldots$ & $\ldots$ & $\ldots$ & $\ldots$ & $\ldots$ & $\ldots$ \\
\hline 4616.09 & -0.117 & $\ldots$ & $\ldots$ & $\ldots$ & $\ldots$ & $\ldots$ & $\ldots$ & $\ldots$ & $\ldots$ & $\ldots$ & $\ldots$ & $\ldots$ & $\ldots$ & $\ldots$ & $\ldots$ & $\ldots$ & $\ldots$ & $\ldots$ & $\ldots$ \\
\hline
\end{tabular}

Notes: A blank indicates that the $E W$ was not reliably measurable, the line was considered blended for the relevant temperature range or yielded a discrepant abundance. The accuracy of the $E W$ measurements is discussed in Sect. 3.

${ }^{a}$ The mean wavelength is given.

${ }^{b}$ Wing of He I $\lambda 4387.9$ taken as pseudo continuum. 